ARTICLE

DOI: $10.1038 / s 41467-018-07058-4$

\title{
Tamoxifen prolongs survival and alleviates symptoms in mice with fatal X-linked myotubular myopathy
}

\author{
Elinam Gayi ${ }^{1}$, Laurence A. Neff (1) ${ }^{1}$, Xènia Massana Muñoz 2,3,4,5, Hesham M. Ismail (1) ${ }^{1}$, Marta Sierra (10 1, \\ Thomas Mercier ${ }^{6}$, Laurent A. Décosterd ${ }^{6}$, Jocelyn Laporte $2,3,4,5$, Belinda S. Cowling (1) $^{2,3,4,5}$, \\ Olivier M. Dorchies (10 ${ }^{1} \&$ Leonardo Scapozza (10) ${ }^{1}$
}

X-linked myotubular myopathy (XLMTM, also known as XLCNM) is a severe congenital muscular disorder due to mutations in the myotubularin gene, MTM1. It is characterized by generalized hypotonia, leading to neonatal death of most patients. No specific treatment exists. Here, we show that tamoxifen, a well-known drug used against breast cancer, rescues the phenotype of Mtm1-deficient mice. Tamoxifen increases lifespan several-fold while improving overall motor function and preventing disease progression including lower limb paralysis. Tamoxifen corrects functional, histological and molecular hallmarks of XLMTM, with improved force output, myonuclei positioning, myofibrillar structure, triad number, and excitation-contraction coupling. Tamoxifen normalizes the expression level of the XLMTM disease modifiers DNM2 and PI3KC2B, likely contributing to the phenotypic rescue. Our findings demonstrate that tamoxifen is a promising candidate for clinical evaluation in XLMTM patients.

\footnotetext{
${ }^{1}$ Pharmaceutical Biochemistry Group, School of Pharmaceutical Sciences, University of Lausanne, University of Geneva, CMU 5-6, Rue Michel-Servet 1, Geneva 1211, Switzerland. ${ }^{2}$ Department of Translational Medicine and Neurogenetics, Institut de Génétique et de Biologie Moléculaire et Cellulaire (IGBMC), IIlkirch 67404, France. ${ }^{3}$ Centre National de la Recherche Scientifique (CNRS), UMR7104, IIlkirch 67404, France. ${ }^{4}$ Institut National de la Santé et de la Recherche Médicale (INSERM), U1258, IIlkirch 67404, France. ${ }^{5}$ Université de Strasbourg, Illkirch 67404, France. ${ }^{6}$ Division and Laboratory of Clinical Pharmacology, Service of Biomedicine, Department of Laboratories, Lausanne University Hospital, Lausanne 1011, Switzerland. These authors contributed equally: Laurence A. Neff, Xènia Massana Muñoz, Hesham M. Ismail. Correspondence and requests for materials should be addressed to

O.M.D. (email: olivier.dorchies@unige.ch) or to L.S. (email: leonardo.scapozza@unige.ch)
} 
X -linked centronuclear myopathy (XLCNM; OMIM \#310400), the most severe form of centronuclear myopathy (CNM), affects $\sim 1$ in 50,000 males $^{1}$. XLCNM is commonly known as X-linked myotubular myopathy (XLMTM) as characteristic histological features include small-caliber myofibers and centrally located nuclei resembling myotubes ${ }^{2,3}$. XLMTM patients present with profound and generalized muscle weakness from birth. Most XLMTM male patients die in the first 2 years of life; some develop a milder form and may survive into adulthood while female carriers display a more heterogeneous disease ${ }^{1,4-6}$. XLMTM is due to the lack of myotubularin (MTM1), a lipid phosphatase that dephosphorylates phosphatidylinositol 3-phosphate (PtdIns(3)P) and PtdIns(3,5) $\mathrm{P}_{2}$ into PtdIns and PtdIns(5) $\mathrm{P}^{7-9}$. Imbalance in these lipids impairs membrane trafficking, nuclei positioning, and t-tubule organization ${ }^{10}$.

No specific treatment exists for XLMTM. AAV-mediated MTM1 replacement therapy showed great promise in murine and canine models and has reached clinical stage (ClinicalTrials.gov Identifier: NCT03199469) ${ }^{11-14}$. Down-regulating dynamin-2 (DNM2), a protein mutated in autosomal forms of CNM, is another promising approach to rescue several CNM forms ${ }^{15-17}$. The development of these innovative therapies, however, may be hampered by technical and safety issues and be further complicated by cost issues in case of clinical efficacy. By contrast, repurposing well-known drugs might offer time- and cost-effective therapeutic options ${ }^{18}$.

Tamoxifen has been used for almost 40 years to treat estrogen receptor-positive breast cancers in both women and men ${ }^{19-21}$ and has been tried in a variety of other disorders ${ }^{22-27}$. Importantly, tamoxifen proved safe in pediatric patients ${ }^{28-35}$. Our earlier published ${ }^{36,37}$ and unpublished findings that tamoxifen potently counteracted the symptoms in a mouse model of Duchenne muscular dystrophy (DMD) have been translated into compassionate use and a phase 3 clinical trial in underway on DMD boys (ClinicalTrials.gov Identifiers NCT02835079 and NCT03354039, respectively).

We thus thought to evaluate tamoxifen in $M t m 1^{-/ y}$ mice, a validated model of XLMTM ${ }^{15,38-40}$. In the present work, we demonstrate that oral tamoxifen given to pups from weaning onwards significantly corrects functional, histological, and molecular hallmarks of the disease, resulting in a remarkable enhancement in survival. Tamoxifen is the first EMA- and FDAapproved drug to show such a promising therapeutic potential for patients with XLMTM.

\section{Results}

Effects of oral tamoxifen treatments on food and drug intake. $M t m 1^{-/ y}$ mice and wild type (WT) littermates were weaned on post-natal day $23 \pm 1$, slightly after disease onset, at which time they were given a control diet or pellets supplemented with tamoxifen citrate (equivalent to 30,10 , and $3 \mathrm{mg}$ tamoxifen free base per kg of diet). Food intake was similar on control and hightamoxifen diets throughout the study time for $M t m 1^{-/ y}$ and wild type mice (Supplementary Figure 1). On the highest dose (30 mg $\mathrm{kg}^{-1}$ of diet), tamoxifen intake was approximately 6,5 , and $4 \mathrm{mg}$ $\mathrm{kg}^{-1} \mathrm{~d}^{-1}$ in treated mice aged 42, 84, and 200 days, respectively. The levels of tamoxifen and major metabolites in plasma and leg muscle tissue were consistent with levels found previously in treated dystrophic mice ${ }^{36}$ (Supplementary Table 1).

Tamoxifen improves life span and slows disease progression. Untreated $M t m 1^{-/ y}$ mice showed early hind limb paralysis with rapid progression of muscle weakness to the trunk and forelimbs, resulting in marked kyphosis, frailty (Fig. 1a) and premature death at a median age of 45 days (Fig. 1b). By contrast, all doses of tamoxifen extended the life span of $M t m 1^{-/ y}$ mice, which on the highest dose, reached a median age of 290 days. Shortly before dying, all untreated $M t m 1^{-/ y}$ mice showed paralyzed hindlimbs and dragging feet. By contrast, adult-treated mice remained mobile and retained ability to rear and to climb onto objects using both forelimbs and hindlimbs (Supplementary Movies 1-4). Remarkably, one $M t m 1^{-/ y}$ mouse on high tamoxifen reached 464 days of age. Consistent with tamoxifen-mediated enhanced survival, disease progression was significantly delayed in treated $M t m 1^{-/ y}$ mice (Fig. 1c), which performed almost as well as WT mice in an anti-gravity assay assessing whole-body force (Fig. 1d). Old $M t m 1^{-/ y}$ mice on high tamoxifen diet presented less clinical features of disease and relatively spared upper body (Fig. 1a), suggesting slower retrograde disease progression. This was illustrated by the ability of the treated mice to climb onto objects using their forelimbs, preserved neck and head control, and relatively large thoracic cage suggestive of spared trunk muscles (Supplementary Movies 3, 4; Fig. 1a). In spite of overwhelming protection from the disease, tamoxifen-treated $M t m 1^{-/ y}$ mice remained small (Fig. 1e). Similarly, tamoxifen-treated WT mice were smaller than their untreated counterparts, partly due to smaller muscles (Supplementary Tables 2, 3), and presumably also because of reduced amount of white adipose tissue, which was evident upon dissection and similar to our observation in dystrophic mice reported earlier ${ }^{36}$.

In order to examine the impact of tamoxifen on muscle mechanical properties, muscle structure and molecular adaptation underpinning tamoxifen-mediated protection, we next treated mice for various periods with optimal tamoxifen dosing (30 $\mathrm{mg} \mathrm{kg}^{-1}$ of diet).

Tamoxifen improves the size of leg muscles and diaphragm. Compared with WT mice, most locomotor muscles examined in $M t m 1^{-/ y}$ mice showed severe atrophy at D42 (Supplementary Table 2,3 ). That atrophy was partly prevented by tamoxifen even after normalization for improved body weight (from $+10 \%$ in the quadriceps to $+18 \%$ in the gastrocnemius). Overall, the effect of tamoxifen at increasing muscle size of $M t m 1^{-/ y}$ mice persisted until D210. The diaphragm of $M t m 1^{-/ y}$ mice showed a distinct response to tamoxifen: although the diaphragm was atrophic at D42 and D84, its absolute size doubled between D84 and D210. As a consequence of changes in body weight, the relative size of the diaphragm was gradually bigger over time (from 67 to 198\% of wild-type counterparts at D42 and D210, respectively).

Tamoxifen improves the strength and features of leg muscles. Muscle weakness is a key pathological feature of XLMTM patients that is also prominent in the $M t m 1^{-/ y}$ murine model. We investigated if and to what extent tamoxifen ameliorated the force generated by the triceps surae (hereafter referred to as triceps), a large muscle group of the lower leg that makes up most of the calf volume and contains gastrocnemius, plantaris, and soleus muscles.

We first analyzed phasic (also known as twitch) force at D42, at which time a substantial proportion of untreated $M t m 1^{-/ y}$ mice survived. The absolute phasic force of untreated $M t m 1^{-/ y}$ mice was highly compromised, representing only $17.3 \%$ of that of untreated wild-type mice. Tamoxifen significantly enhanced the absolute force of $M t m 1^{-/ y}$ mice almost threefold (Fig. 2a). The absolute phasic traces were normalized to the triceps crosssectional area in order to express the specific phasic tension, i.e., the force generated per unit of muscle cross-section (Fig. 2b). This revealed that tamoxifen markedly augmented the specific phasic force of $M t m 1^{-/ y}$ mice $(+155 \%)$, reaching $61 \%$ of the force developed by WT mice. We then compared the specific phasic force at D42, D84, and D210 (Fig. 2c). Tamoxifen 
a

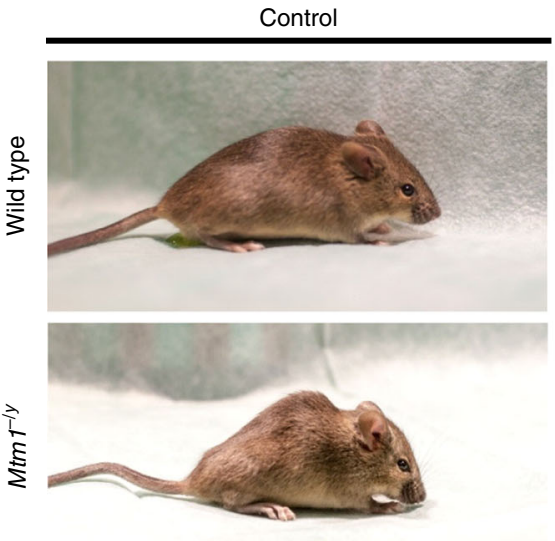

Young (42 days)

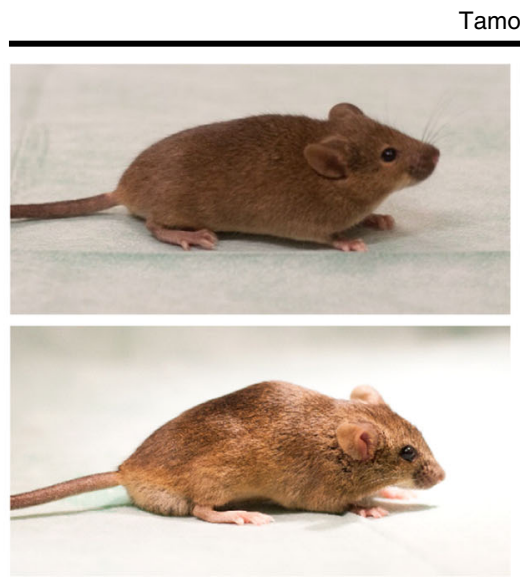

Adult (80 days) amoxifen

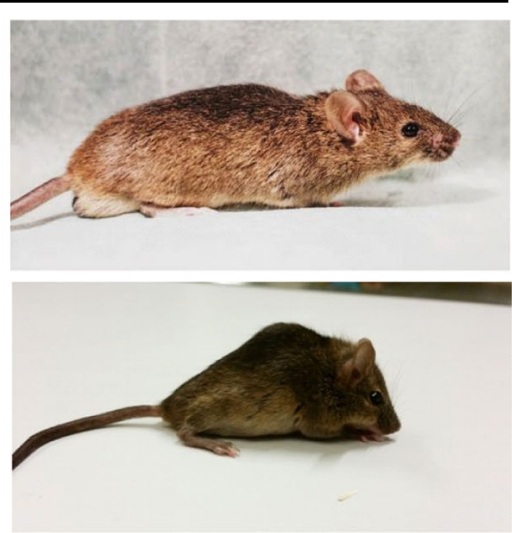

Old (>200 days) b

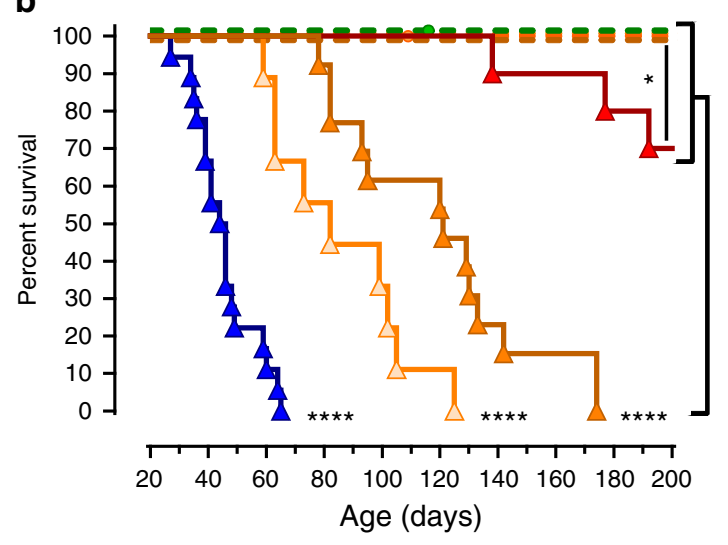

C

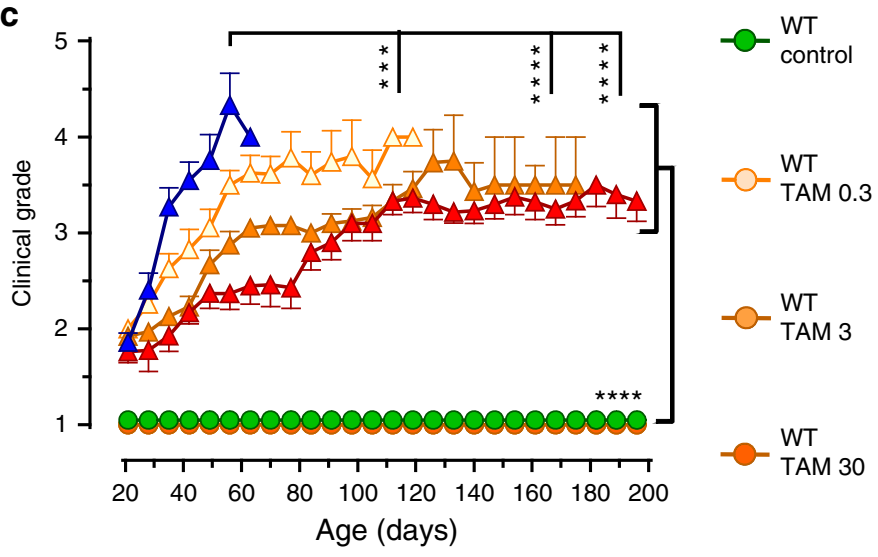

d

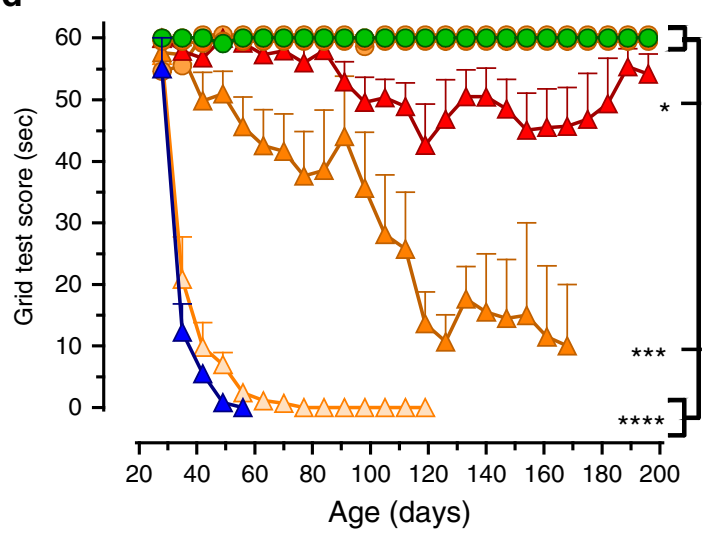

e

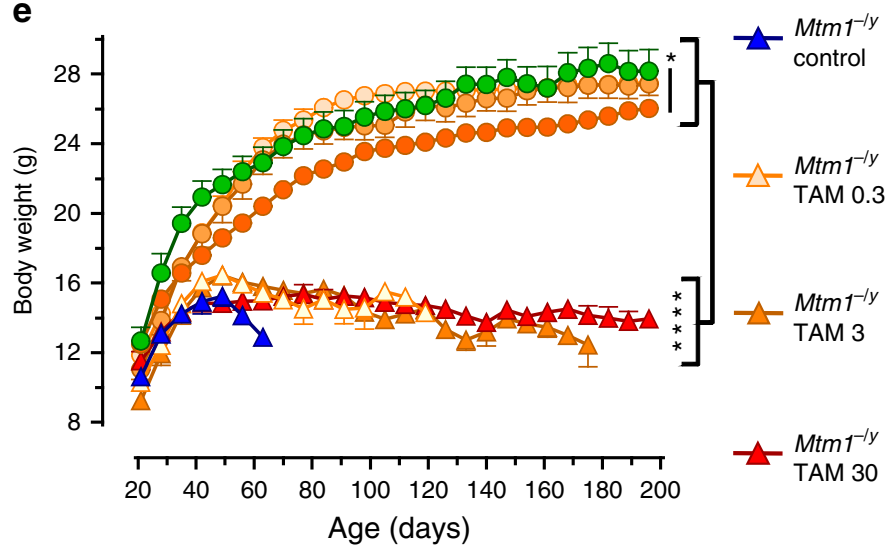

Fig. 1 Tamoxifen rescues the phenotype of XLMTM mice. Life-long oral tamoxifen delays disease progression in XLMTM mice. Wild type (WT) and $\mathrm{Mtm}^{-/ \mathrm{y}}$ mice were fed control or tamoxifen (TAM)-supplemented diets from weaning onward. a Photographs of WT and Mtm1-/y mice, illustrating disease severity in young untreated mice and protection conferred by tamoxifen in adult and old mice. $\mathbf{b}$ Kaplan-Meier curves showing the effects of treatments on mouse survival. Early death of untreated $M t m 1^{-/ y}$ mice contrasts with prolongation of life span in tamoxifen-treated littermates. Data are from 8 to 18 mice per group. ${ }^{\star} P \leq 0.05 ;{ }^{\star \star \star \star} P \leq 0.0001$. Log-rank (Mantel-Cox) test. c Disease progression was assessed three times per week using a 5-grades clinical scale: muscle function was scored as 1 (normal function of hind limbs), 2 (difficulty in spreading toes), 3 (evident weakness in legs), 4 (paralysis of one hind limb), or 5 (complete paralysis of both legs). WT mice had a clinical score of 1 (normal) throughout the study. Untreated Mtm1-/y mice quickly reached a high clinical grade, whereas disease progressed much slower in tamoxifen-treated littermates. $\mathbf{d}$ Mouse motor function was assessed weekly via a horizontal grid-hanging test. The score of untreated Mtm1-/y mice declined quickly. Tamoxifen preserved motor function of $M t^{-1 m} 1^{-/ y}$ mice close to WT values. e Mouse body weight was recorded 3 times per week. Tamoxifen affected the growth of WT but not of Mtm $1^{-/ y}$ mice. Mtm $1^{-/ y}$ mice remained smaller than WT mice throughout. b-e Symbols and TAM doses $\left(0.3,3\right.$, and $30 \mathrm{mg} \mathrm{kg}^{-1}$ of diet) are shown on the right-hand side. $\mathbf{c}$, $\mathbf{d}$, e Data represent the mean \pm s.e.m. of 8 to 18 mice as defined in $\mathbf{a} .{ }^{\star} P \leq 0.05 ;{ }^{\star \star} P \leq 0.01 ;{ }^{\star \star \star \star} P \leq 0.0001$; ns non-significant. One-way ANOVA with Fisher's least significance difference (LSD) post-test 

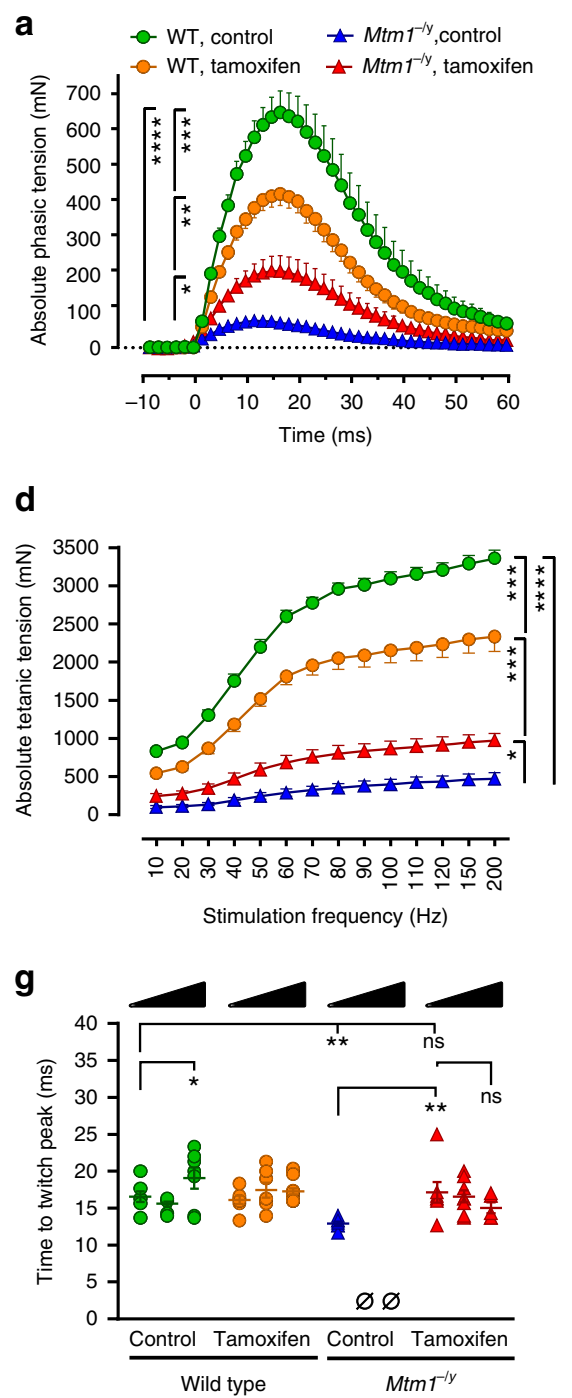

b

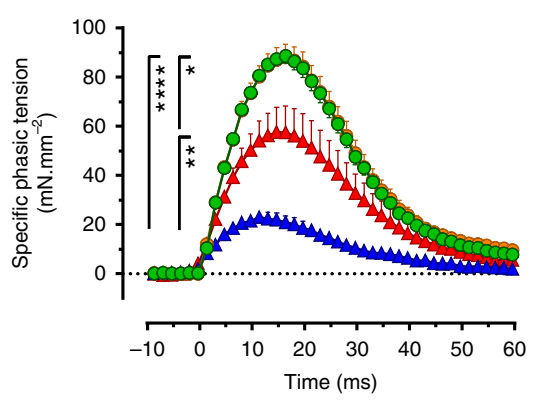

e

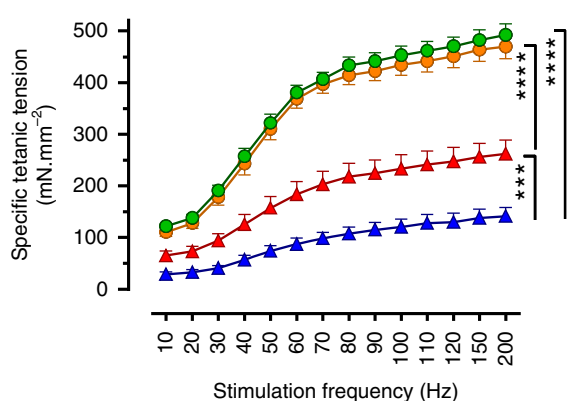

h

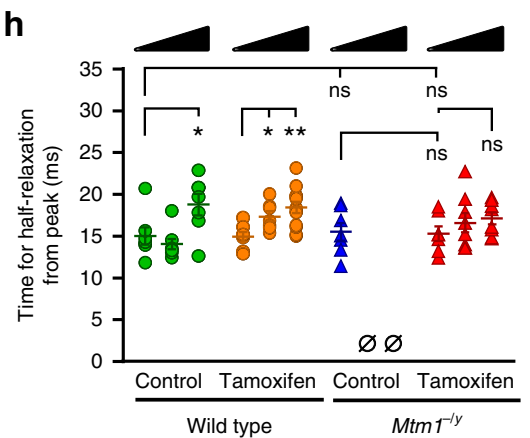

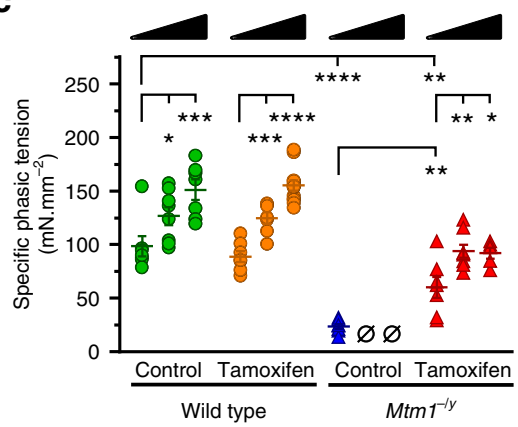

f

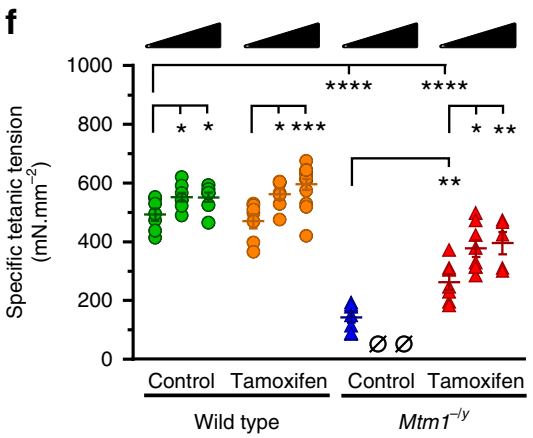

Fig. 2 Tamoxifen augments the strength of leg muscles of XLMTM mice. Electrically evoked triceps contractions were recorded under isometric conditions and contractile features analyzed in 42, 84, and 210-day-old mice fed placebo or tamoxifen-supplemented (30 $\left.\mathrm{mg} \mathrm{kg}^{-1}\right)$ diets. a Average traces showing absolute phasic (twitch) contraction of the triceps at 42 days (D42). Tamoxifen significantly enhanced the force (tension) in $M$ tm $1^{-/ y}$ mice. $\mathbf{b}$ Specific phasic force (see Online Methods for details) of D42 Mtm1-/y mice augmented with tamoxifen. c Specific phasic force of $M t m 1^{-/ y}$ mice increased with tamoxifen at all examined ages. d Average traces showing triceps absolute force-frequency curves at D42. Tamoxifen more than doubled the tetanic force of $M t m 1^{-/ y}$ mice. e Specific force of $M t m 1^{-/ y}$ mice markedly augmented with tamoxifen at all stimulation frequencies. f Specific tetanic force of $M t m 1^{-/ y}$ mice was considerably improved at all studied ages. In triceps examined after various treatment durations, tamoxifen rescued the impaired time to twitch peak of $\mathrm{Mtm} 1^{-/ y}$ mice (g) but had no impact on the time required for half-relaxation from peak (h). D42 panels: WT wild type; legend in a stands for $\mathbf{a}, \mathbf{b}, \mathbf{d}$, e; data represent the mean \pm s.e.m. of $n=7$ triceps per group. $\mathbf{c}, \mathbf{f}, \mathbf{g}$, $\mathbf{h}$ : black triangles illustrate increasing age ( $42-84$ and 210 days) within each treatment group; columns from left to right: data represent the mean \pm s.e.m. of $7 ; 8 ; 7 ; 7 ; 7 ; 13 ; 7 ; 0 ; 0 ; 7 ; 8 ; 6$ triceps, respectively. ${ }^{\star} P \leq 0.05 ;{ }^{\star \star} P \leq 0.01$; ${ }^{\star \star \star} P \leq 0.001$; ${ }^{\star \star \star \star} P \leq 0.0001$; ns non-significant, $\varnothing$ no surviving mice. One-way ANOVA followed by Fisher's LSD post-test

considerably increased this parameter in $M t m 1^{-/ y}$ mice at all ages studied compared to untreated $M t m 1^{-/ y}$ mice at D42 (Fig. 2c). Functional benefits culminated to $74.1 \%$ of WT values at D84 and showed only marginal decrease at D210.

Then, the tetanic forces were determined from force-frequency curves. Tamoxifen more than doubled (2.1-fold increase) the absolute tetanic force of $M t m 1^{-/ y}$ mice at D42, which, after correction for muscle size, reached $53.2 \%$ of WT values (Fig. $2 \mathrm{~d}$, e). Similar to phasic force, tamoxifen remarkably increased the specific tetanic force of $M t m 1^{-/ y}$ mice at all ages studied (Fig. 2f). Maximum benefits were found at D210, when tetanic force reached $71.7 \%$ of age-matched wild type values $(68.2 \%$ at D84).

Further analysis of the phasic traces revealed a significant reduction in the time required by the triceps of untreated
$M t m 1^{-/ y}$ mice for reaching twitch peak force, indicative of impaired excitation-contraction coupling (Fig. 2g). Tamoxifen fully restored the time to twitch peak to normal values at all ages. Tamoxifen did not significantly alter the time for half-relaxation from twitch peak at D42 (indicative of SERCA activity and kinetics of inactivation of actin-myosin cross-bridges) (Fig. 2h). Nevertheless, this feature tended to increase with age in both WT and $M t m 1^{-/ y}$ mice treated with tamoxifen.

In striking contrast with its beneficial actions in $M t m 1^{-/ y}$ mice, tamoxifen caused generalized muscle hypotrophy in WT mice, which was only partly explained by decreased body weight (Supplementary Table 2, 3). In WT triceps at D42, this translated into decreased absolute force output (Fig. 2a, d), which correlated with decreased muscle mass (Fig. 2b, e). However, specific force 
was not altered suggesting that intrinsic mechanical properties of WT myofibers were globally preserved. The specific phasic and tetanic tensions of WT mice augmented with age independently of tamoxifen (Fig. 2c, f).

Tamoxifen improves leg muscle structure and ultrastructure. Histological analyses showed that the tibialis anterior (TA) muscle of $M t m 1^{-/ y}$ mice contained many fibers in which organelles, including nuclei and mitochondria, were abnormally distributed, forming necklace patterns of oxidative staining essentially in large-caliber fibers ${ }^{41}$. Tamoxifen did not correct these specific features and had a minor impact on fiber type composition (Fig. 3a, b; Supplementary Figure 2). However, tamoxifen reduced by $\sim 50 \%$ the number of myofibers displaying nuclei in abnormal position, a prominent histological feature of XLMTM (Fig. 3c). Tamoxifen also induced overall improvement of the myofibrillar structure, including improved organization of the Z-lines, highly ordered protein complexes that keep the sarcomeres in register, and better defined M-line, A-band, and Iband (Fig. 3d; Supplementary Figure 3).

Tamoxifen reduces disease modifiers and alters ER levels. We examined the consequence of tamoxifen treatment on muscular transcript and protein levels of targets selected for their known contribution to $\mathrm{CNM}$ pathogenesis, for modulating $\mathrm{Mtm} 1^{-/ \mathrm{y}}$ mouse phenotype, or mediating tamoxifen actions (Fig. 4; Supplementary Tables 4,5$)$. In the $M t m 1^{-/ y}$ mouse, as well in CNM patients, both BIN1 and DNM2 are overexpressed. Here we report that BIN1 mRNA is also more abundant in $M t m 1^{-/ y}$ mouse muscle. Overall, in both WT and $M t m 1^{-/ y}$ gastrocnemius muscles, tamoxifen tended to down-regulate BIN1 and DNM2 (Fig. $4 \mathrm{a}-\mathrm{c}$ ), both postulated to act downstream of myotubularin ${ }^{42}$. Of note, the ability of tamoxifen to reduce DNM2 levels was reproduced, at least partly, in a human muscle cell line established from an XLMTM individual ${ }^{43,44}$ (Supplementary Figure 4; see Supplementary Tables 6, 7 for details on the human muscle cell lines and the primers used for RT-PCR). In mice, tamoxifen also significantly corrected the elevated levels of desmin (a musclespecific intermediate filament that controls nuclei positioning via binding myotubularin ${ }^{45}$ ), of PI3KC2B (an enzyme whose genetic muscle-specific ablation rescues the disease ${ }^{40}$ ) and of dysferlin (involved in membrane repair and t-tubule biogenesis ${ }^{46,47}$ ) (Fig. $4 \mathrm{a}, \mathrm{b}, \mathrm{d}$ ). Muscle extracts from untreated $M t m 1^{-1 y}$ mice massively accumulated a putative dysferlin degradation product $(\sim 160 \mathrm{kDa})$ whose proportion was reduced to near-normal levels with tamoxifen (Supplementary Figure 5). Tamoxifen decreased ER $\alpha$ but not ER $\beta$ protein levels, presumably via posttranscriptional mechanisms since these effects were not mirrored by mRNA expression (Fig. 4a, b, e). Tamoxifen also partly restored sarcomeric myosin content, which was significantly reduced in $M t m 1^{-/ y}$ muscle (Fig. 4b, f).

Tamoxifen increases triad density and restores EC coupling. Well-formed triads are required for efficient calcium release from intracellular stores and subsequent muscle contraction in response to nerve stimulation. Tamoxifen restored triad quantity and improved their morphology (Fig. 5a, b). Consistent with these findings, tamoxifen normalized the amplitude of calcium fluxes elicited by depolarization in myofibers from $M t m 1^{-/ y}$ mice (Fig. 5c-e). Moreover, tamoxifen almost corrected the levels of DHPR, a voltage-sensitive calcium channel that tightly regulates excitation-contraction (EC) coupling. The DHPR effector, RyR1, was found at normal levels in all groups (Fig. $5 f-g$ ).
Long-term tamoxifen mitigates myopathic feature progression. TA muscles of $M t m 1^{-/ y}$ mice treated until D84 and D210 still contained small caliber myofibers with abnormally distributed organelles (Fig. 6a, b). The diameter of TA myofiber significantly increased with age in WT mice while it remained virtually stable over time in tamoxifen-treated $M t m 1^{-/ y}$ mice (Fig. 6c). At D42, tamoxifen more than halved myofibres with nuclei in abnormal position in $M t m 1^{-/ y}$ mice. Then, these pathological myofibres gradually accumulated as treated $M t m 1^{-/ y}$ mice became older (Fig. 6d). At D42, tamoxifen reduced by $42 \%$, the deficit in triad number of $M t m 1^{-/ y}$ mice. Between D42 and D210, triad number declined similarly in both WT and $M t m 1^{-/ y}$-treated mice (Fig. 6e).

\section{Discussion}

XLMTM is a rare myopathy due to the lack of the lipid phosphatase myotubularin (MTM1) ${ }^{1,48}$. Most affected boys die during early infancy ${ }^{6}$. Despite extremely high unmet medical need, very few pharmacological options have been explored so far in animal models $^{49,50}$ or in CNM patients ${ }^{51}$.To date, no approved pharmacological treatment has been shown to alleviate the symptoms and increase the life expectancy of the patients.

The rationale for repurposing tamoxifen for XLMTM was based on previous success of our group with the drug in several murine models of debilitating muscular diseases, including $m d x^{5 C v}$ mice, a model of $\mathrm{DMD}^{36}$. We reasoned that the many protective actions exerted by tamoxifen on diverse muscular conditions might provide a therapeutic avenue for XLMTM. Our findings largely support this view.

In brief, oral tamoxifen, administered from shortly after weaning (i.e., after $M t m 1^{-/ y}$ mice started to develop overt muscular symptoms), improved overall body strength, increased force generation in leg muscles, slowed disease progression through clinical stages, and importantly, prolonged the lifespan severalfold. These functional improvements correlated with reduced number of centrally located myonuclei, better excitation-contraction coupling, and normalization of molecular markers of the disease, all of which likely contribute to tamoxifenmediated protection.

Efficient transduction of the muscular action potential into release of $\mathrm{Ca}^{2+}$ from sarcoplasmic reticulum stores is a critical step in excitation-contraction coupling (ECC) and a major determinant of force generation. Triads are key structures in this process. Triad disorganization and impaired ECC have a major role in the profound muscle weakness displayed by $M t m 1^{-/ y}$ mice and is associated with a decreased level of DHPR ${ }^{52-55}$. Tamoxifen not only restored about $42 \%$ of triad number deficit in the TA, but also almost fully normalized DHPR expression and dramatically enhanced $\mathrm{Ca}^{2+}$ release in isolated flexor digitorum brevis (FDB) fibers exposed to a physiological stimulus that triggers DHPR-mediated RyR activation. Altogether, beneficial actions of tamoxifen on triad count, structural components, and function very likely explain the much improved muscle function of $M t m 1^{-/ y}$ mice. The augmented muscle function was measured not only locally in the triceps, but also at the whole body level with the grid hanging test, suggesting that tamoxifen-mediated enhancement of ECC took place in the whole musculature. Moreover, $M t m 1^{-/ y}$ diaphragm weight was gradually increased over time.

Noteworthy, although body weight deficit was not corrected and improved muscle structure and muscle force did not reach WT level, the lifespan of tamoxifen-treated $M t m 1^{-/ y}$ mice was extended by 6.4 -fold ( 45 days median survival of untreated $\mathrm{KO}$ vs 290 days) on high dose tamoxifen. Overall, it suggests that the beneficial effects of tamoxifen on life-sustaining physiological 
a
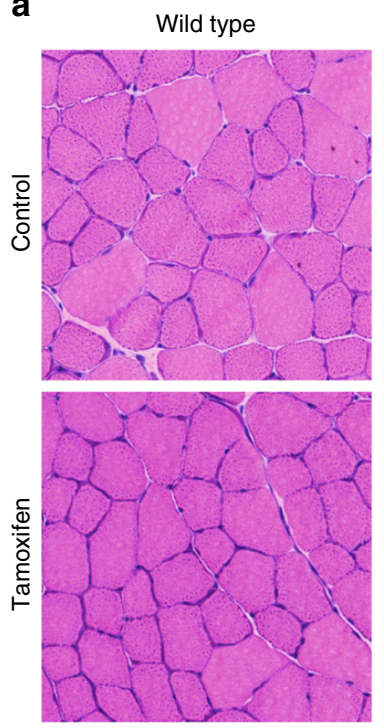

C

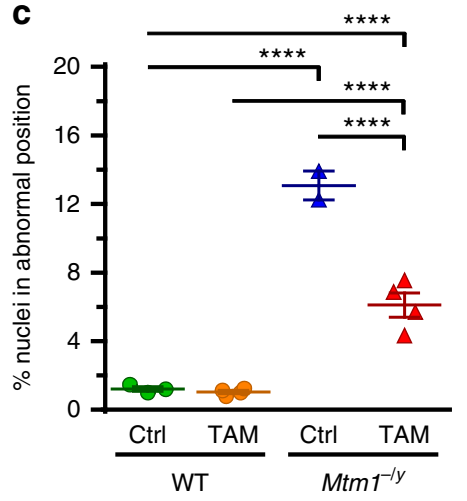

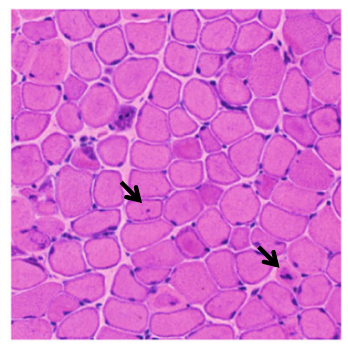

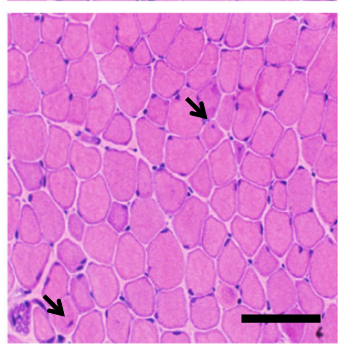

b

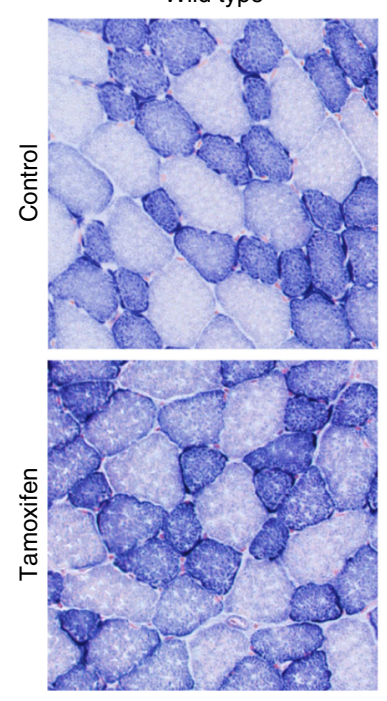

$M t m 1^{-1 y}$

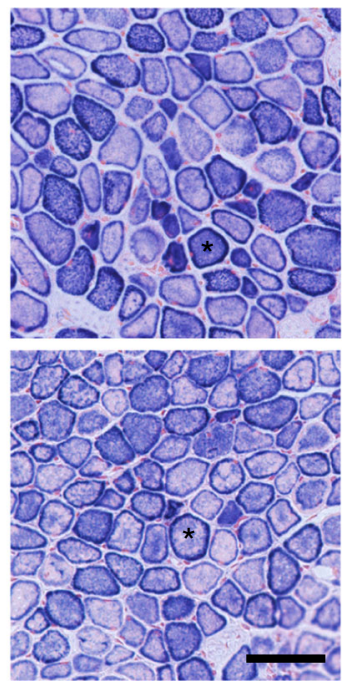

d
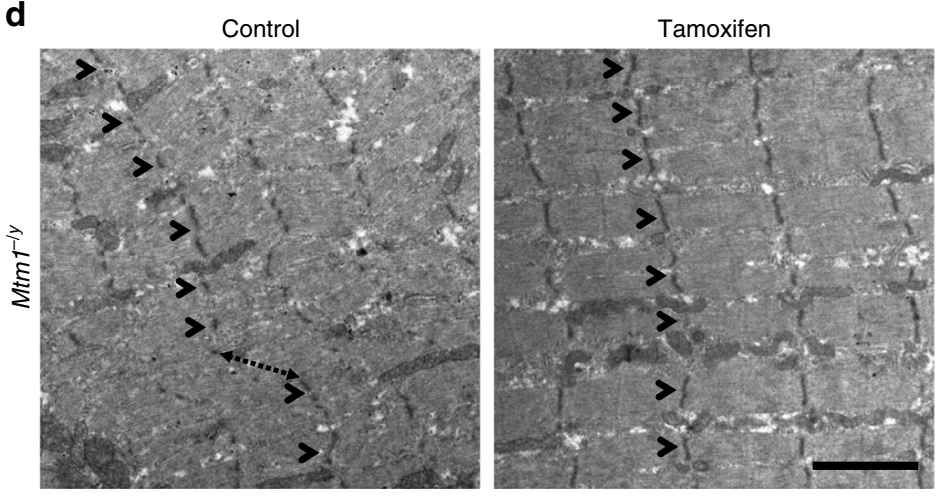

Fig. 3 Tamoxifen mitigates muscle structure and improves sarcomeric organization in XLMTM mice. Muscle structure and sarcomere ultrastructure were examined by histology and transmission electron microscopy, respectively, in 42-day-old (D42) mice either untreated (control; Ctrl) or treated with tamoxifen (TAM; $30 \mathrm{mg} \mathrm{kg}^{-1}$ of diet). a Representative pictures of hematoxylin-eosin stained sections from the tibialis anterior (TA) of D42 mice. Note the small size of the $M t m 1^{-/ y}$ myofibres and the presence of mislocalized nuclei, a hallmark of $M t m 1^{-/ y}$ mice and XLMTM patients (arrows). $\mathbf{b}$

Representative pictures of succinate dehydrogenase (SDH) activity in TA sections of D42 mice, demonstrating abnormal distribution of oxidative staining. The intense staining forming a ring at the periphery of many $M t m 1^{-/ y}$ myofibres (examples shown by asterisks) is due to accumulated mitochondria and other organelles and is a hallmark of the pathology. c The percentage of nuclei abnormally positioned (either internally or centrally located) in TA myofibres of D42 mice were counted from hematoxylin-eosin-stained sections. That feature was reduced by $53.3 \%$ with TAM. Data represent the mean \pm s.e.m. of $n=2-4$ TA per group. ${ }^{\star \star \star \star} P \leq 0.0001$. One-way ANOVA followed by Fisher's LSD post-test. $\mathbf{d}$ Sarcomere ultrastructure revealed by transmission electron microscopy in the TA from untreated (control) and tamoxifen-treated Mtm1-/y mice at D42. Note overall disorganization of the sarcomeres in untreated TA with disruption of the Z-lines (structures running perpendicular to the sarcomeres and holding myofibrils together; arrowheads) and shifted sarcomeres (dashed arrow). Tamoxifen ameliorated the organization of the sarcomeres as demonstrated by in-register Z-lines in adjacent myofibrils (arrowheads). The bar represents $50 \mu \mathrm{m}$ in $\mathbf{a}$ and $\mathbf{b}$, and $2 \mu \mathrm{m}$ in $\mathbf{d}$

functions are sufficient to greatly extend the lifespan of the animals. Most morphological, functional, and histological outcomes were stable until around 210 days of age. Preliminary observation of long-term $\mathrm{Mtm}^{-/ \mathrm{y}}$ mouse survivors (4 mice; average 369 days; range 271-464 days; i.e., up to 10-fold the median survival of untreated mice) suggested that the beneficial effects of tamoxifen persisted on extended periods of time.

Here we also investigated potential molecular mechanisms of the rescue. Our earlier work with tamoxifen in $m d x^{5 C v}$ mice $^{36}$ provided evidence that tamoxifen (i) is pro-estrogenic on striated muscles (as on bone and uterus ${ }^{56}$ ), a view supported by a recent meta-analysis in treated women ${ }^{57}$, and (ii) exerts beneficial actions through high affinity estrogen receptors. Typical of hormone-dependent receptor degradation, binding of tamoxifen (or its active metabolites) to ERa likely explains the lower levels of that receptor in muscles of treated $M t m 1^{-1 y}$ mice mice ${ }^{58-60}$.
Myotubularin is intricately linked to BIN1 and DNM2, which collectively control membrane dynamics from production of phosphoinositides to membrane curvature and vesicle fission ${ }^{42}$. Noteworthy, mutations in BIN1 and DNM2 cause autosomal forms of $\mathrm{CNM}^{42}$. MTM1 and BIN1 were proposed to act as negative regulators of DNM2 in muscle. Accordingly, diminishing DNM2 rescues the phenotype of $M t m 1^{-1 y}$ and $B i n 1^{-1-}$ mice ${ }^{15-17}$. We found that tamoxifen almost normalized BIN1 and DNM2. On their own, these molecular actions taking place just downstream of MTM1 might explain most tamoxifen-driven benefits. Furthermore, we showed that tamoxifen also reduced DNM2 levels in a human muscle cell line established from an XLMTM individual ${ }^{43,44}$. This further demonstrates the value of the $M t m 1^{-/ y}$ mouse as a model for XLMTM ${ }^{15,38-40}$ and supports future translation of tamoxifen efficacy findings from $M t m 1^{-/ y}$ mice to XLMTM patients. 
a
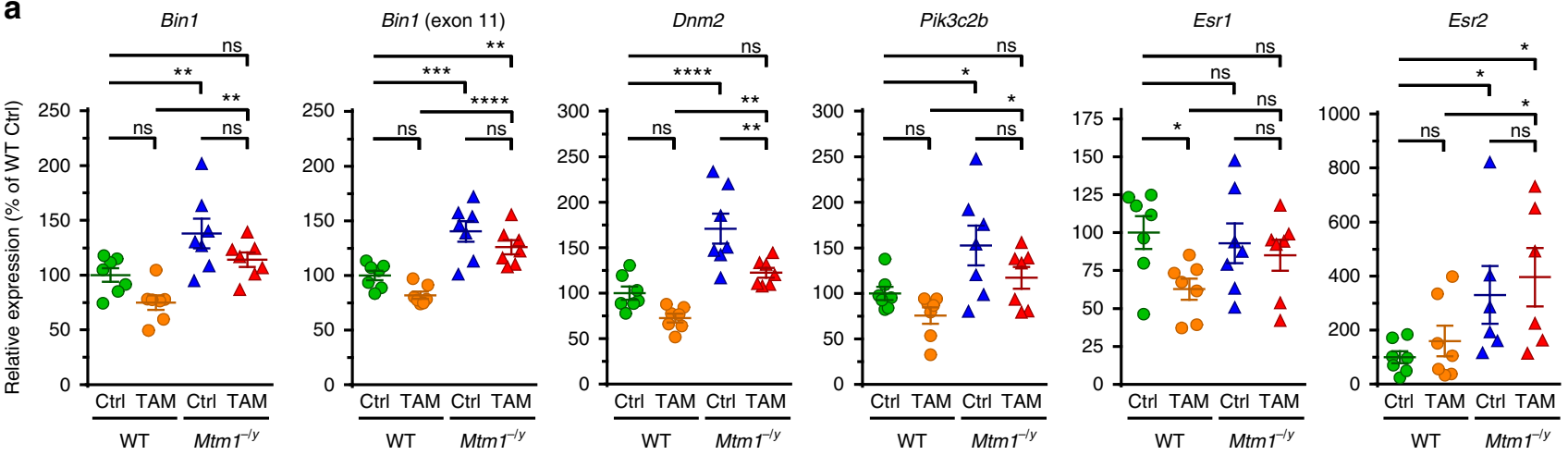

b

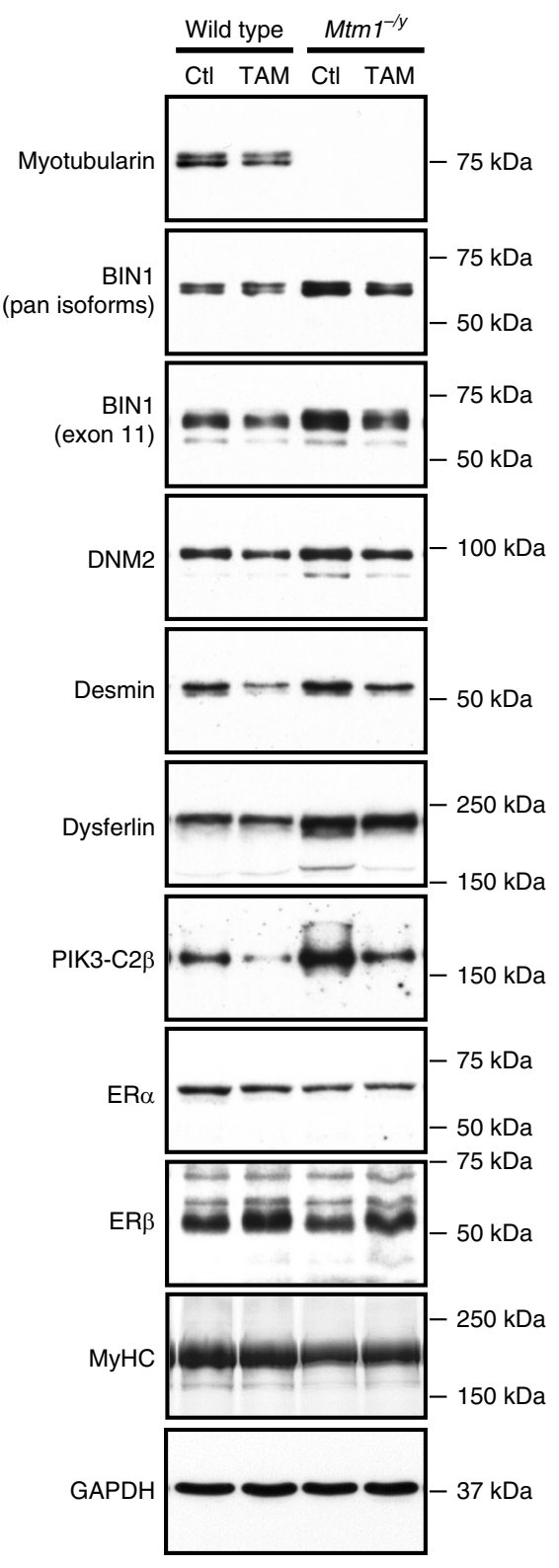

C

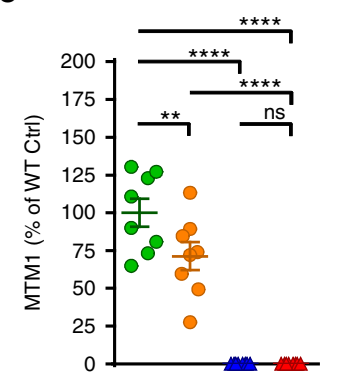

d

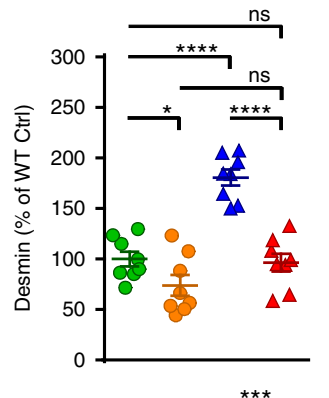

e
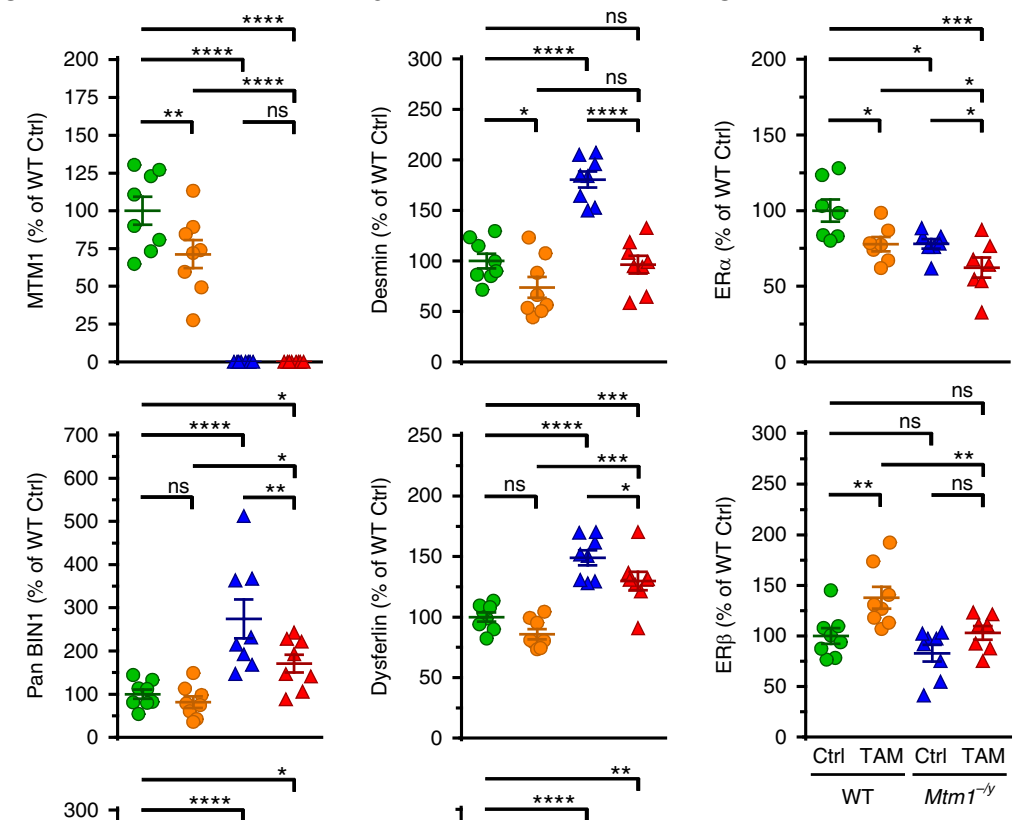
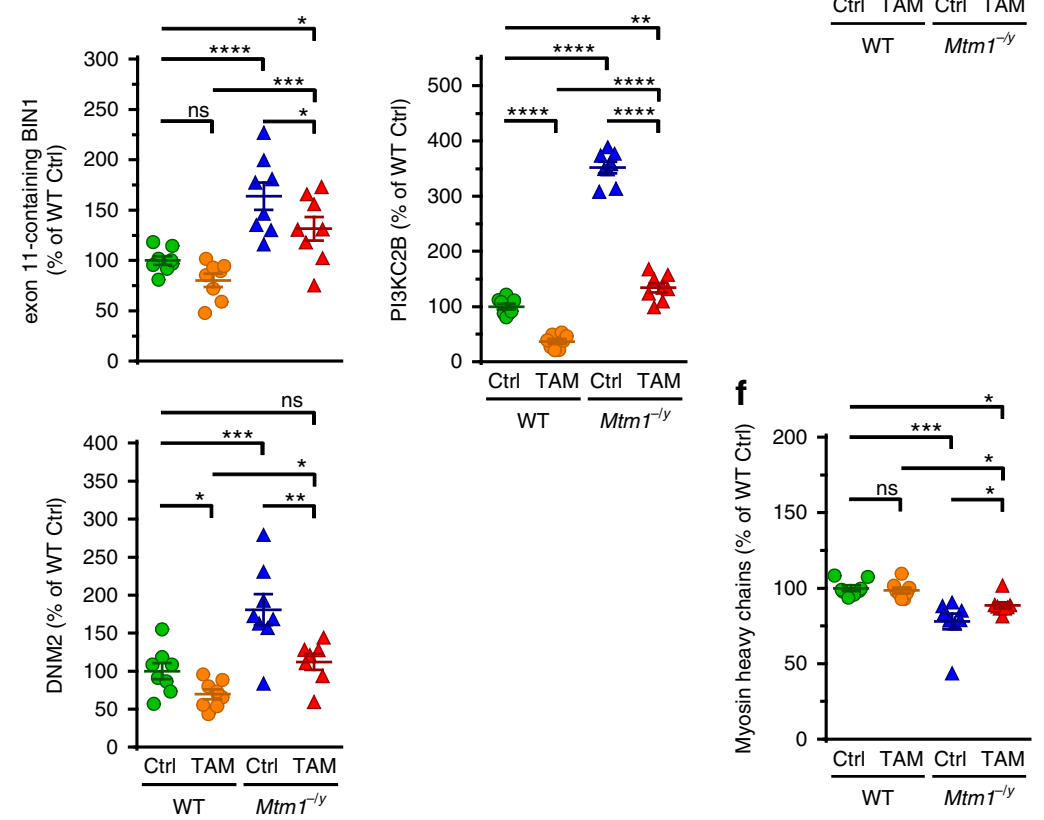

Interestingly, a recent study in MCF7 cells linked DNM2 reduction to impaired autophagy-a prominent feature in $M t m 1^{-/ y}$ mice 39,61 and to ERa accumulation in response to estradiol $^{62}$. Although impaired autophagy have been reported in $M t m 1^{-/ y}$ mice, DNM2 levels are usually increased (and not reduced) in XLMTM and relevant model organisms. In line with these reports, our observations suggest that other mechanisms prevail in $M t m 1^{-/ y}$ muscle since tamoxifen (i) decreased not only DNM2 but also ERa levels, and (ii) improved overall muscle architecture and nuclei positioning, hardly consistent with further 
Fig. 4 Tamoxifen reduces the expression of XLMTM disease modifiers and alters the expression of estrogen receptors. The mRNA and protein levels of known XLMTM disease modifiers and of estrogen receptors, which mediate most tamoxifen actions, were determined in gastrocnemius muscle of 42 -days old (D42) wild type (WT) and Mtm1-/y mice, either untreated (control; Ctrl) or tamoxifen (TAM)-treated (30 mg kg ${ }^{-1}$ of diet). a mRNA levels normalized to glyceraldehyde-3-phosphate dehydrogenase (Gapdh). From left to right, relative expression (as percentage of WT Ctrl) of mRNA encoding amphiphysin 2/BIN1 (Bin1; all transcripts), muscle-specific BIN1 (Bin1; exon 11-containing transcript), dynamin-2 (Dnm2), PI3KC2B (Pik3c2b), estrogen receptor (ER) $\alpha$ (Esr1), and ERR (Esr2). b Representative immunoblots of proteins of interest, as indicated. DNM2: dynamin 2; MyHC: myosin heavy chains; GAPDH: glyceraldehyde-3-phosphate dehydrogenase. Position of molecular weight markers $(k D a)$ is shown. $\mathbf{c}-\mathbf{f}$ Levels (normalized to GAPDH and expressed as percentage of WT Ctrl) of proteins selected for their role in XLMTM and tamoxifen signaling. c Proteins involved in the "MAD"-pathway. From top to bottom: myotubularin (MTM1), amphiphysin 2/BIN1 (pan-isoforms), amphiphysin 2/BIN1 (muscle-specific isoform), dynamin-2 (DNM2). d Other disease modifiers and protein deregulated in absence of MTM1. From top to bottom: desmin, dysferlin, PI3KC2B. e Estrogen receptors. Top: ER $\alpha$; bottom: ER $\beta$. $\mathbf{f}$ Myosin heavy chains, a major constituent of sarcomeres. Data represent the mean \pm s.e.m. of $n=6-8$ muscles per group. ${ }^{\star} P \leq 0.05 ;{ }^{\star \star} P \leq 0.01$; ${ }^{\star \star \star} P \leq$ $0.001 ;{ }^{\star \star \star \star} P \leq 0.0001 ;$ ns non-significant. One-way ANOVA followed by Fisher's LSD post-test

worsening of autophagic flux subsequent to DNM2 downregulation.

Phosphatidylinositol 3-phosphate (PtdIns3P) is a main substrate of myotubularin and is produced by PI3-kinases including class II PI3KC2B. Consistent with the hypothesis that altered PtdIns3P level account for myotubular myopathy, recent studies showed that inhibiting or down-regulating PI3KC2B rescued the phenotype of $M t m 1^{-/ y}$ mice, likely via improvement of $\mathrm{ECC}^{40,55}$. The PI3KC2B kinase was over-expressed several-fold in $M t m 1^{-/ y}$ gastrocnemius muscle. Remarkably, we found that tamoxifen almost normalized PI3KC2B expression, thereby very likely contributing to the overall protection exerted by tamoxifen. It is not established, however, if the reduced levels of PI3KC2B is a direct effect of tamoxifen or if it is a secondary consequence of an overall improvement of muscle structure and function. Of note, our findings on PI3KC2B levels diverge from those by Dr. James Dowling and colleagues (see ref. ${ }^{63}$ ), suggesting they might be dependent on the mouse genetic background and/or disease severity.

Desmin is a muscle-specific intermediate filament that shows discrete sarcomeric expression. It helps maintaining the myofibrils in-register and ensuring mechanical integrity of the myofibers during contraction. In $M t m 1^{-/ y}$ muscle and in XLMTM patients, desmin level is increased ${ }^{45,64}$ and its distribution resembles that in immature myotubes ${ }^{44,64}$. Desmin also controls nuclei and mitochondria positioning, a function that is altered by mutations in $M T M 1^{45}$. We showed here that tamoxifen normalized the elevated levels of desmin in $M t m 1^{-1 y}$ mice and this correlated with improved nuclei position and better sarcomeric structure, along with partial correction of myosin heavy chain levels, the major component of myofibrils.

Although tamoxifen significantly reduced DNM2, PI3KC2B and desmin in muscle of both $\mathrm{WT}$ and $M t m 1^{-/ y}$ mice, these molecular changes led to dramatically different consequences. In $M t m 1^{-/ y}$ mice, normalization of elevated levels of these proteins likely improved the phenotype. By contrast, muscle growth and function in normal mice was impaired with tamoxifen. We speculate that this is a consequence of the reduction of these proteins and of MTM1 to sub-optimal levels. Considering the roles of the MTM1-BIN1-DNM2 pathway in t-tubule biogenesis and in excitation-contraction coupling $17,52,53,65$, it is possible that muscle cramps experienced by breast cancer patients ${ }^{66-68}$ result from tamoxifen-induced changes in the levels of these proteins. By contrast, we believe that tamoxifen-induced muscle atrophy observed in WT mice is likely species-specific as, to our knowledge, alteration of muscle volume has not been reported in human, not even in breast cancer patients having been on tamoxifen for several years.

In this study, we show that tamoxifen, given orally to a mouse model of XLMTM, considerably ameliorated muscle function and prolonged survival. As reported earlier in a murine model of $\mathrm{DMD}^{36}$, tamoxifen also caused relative hypertrophy of the diaphragm of aged $M t m 1^{-/ y}$ mice, which warrants further investigation for assessing the potential benefit on respiratory function. The most efficacious dose tested $(30 \mathrm{mg}$ of tamoxifen per $\mathrm{kg}$ of diet, yielding 4-6 mg of tamoxifen per $\mathrm{kg}$ of body weight per day) was similar to that producing optimal disease prevention in a mouse model of $\mathrm{DMD}^{36}$. Considering differences in drug exposure between species, this dose is clinically relevant as it matches that used for breast cancer therapy, compassionate use for DMD boys and TAMDMD, the phase 3 clinical trial (respectively ClinicalTrials.gov identifiers NCT02835079 and NCT03354039). Growth pattern in tamoxifen-treated mice was not restored to normal, which should be distinguished from a potential toxicity of tamoxifen on growth. As tamoxifen modulates molecular events downstream of myotubularin, not all pathogenic features were corrected to normal values as would be expected with gene-restoration therapies ${ }^{13}$. Interestingly, tamoxifen has been used as an alternative to growth hormone in short boys $^{31}$ and improved predicted adult height in girls with McCune-Albright syndrome ${ }^{35}$. Thus, side effects related to suboptimal growth of XMLTM patients in future tamoxifen trials are extremely unlikely.

Interestingly, during the course of our study, Professor James Dowling and collaborators (Sick Kids Hospital, Toronto, Canada) made observations similar to ours with tamoxifen in $M t m 1^{-/ y}$ mice, confirming the clinically relevant findings that we present here.

Importantly, tamoxifen is a readily available EMA- and FDAapproved drug used for several decades for treating breast cancer $^{19-21}$. It is also efficacious in other hormone-related disorders $^{22-27}$ and is safe in diverse male pediatric conditions ${ }^{28-35}$. We believe that the Orphan Drug Designation recently granted by the EMA for tamoxifen use in $\mathrm{DMD}^{36,37}$, the launching of the TAMDMD trial, and simultaneous report of the therapeutic potential of tamoxifen in $M t m 1^{-/ y}$ mice by two independent groups should encourage priority consideration for clinical evaluation for XLMTM. Tamoxifen fulfils all criteria ${ }^{18}$ for timely repurposing as a symptomatic treatment for XLMTM, either as a monotherapy or combined with AAV-MTM1 restoration or DNM2 downregulation approaches that will hopefully prove efficacious in clinical trials. Tamoxifen might also prove useful in other myopathies where t-tubule defects secondary to increased DNM2 levels contribute to the pathogenesis, such as BIN1-related CNM and caveolin-3 and dysferlin-related myopathies ${ }^{15,17,65,69}$.

\footnotetext{
Methods

Animals and housing conditions. All procedures involving mice complied with the Swiss Federal Law on Animal Welfare. Procedures were reviewed by the veterinary office of Geneva and authorized under the license numbers GE/61/16 and GE/13/17.

Mtm1-deficient mice in the 129Pas genetic background were obtained from Dr. J. Laporte (IGBMC, Illkirch, France). A colony was established and maintained at the animal facility of the Geneva-Lausanne School of Pharmaceutical Sciences by
} 
a
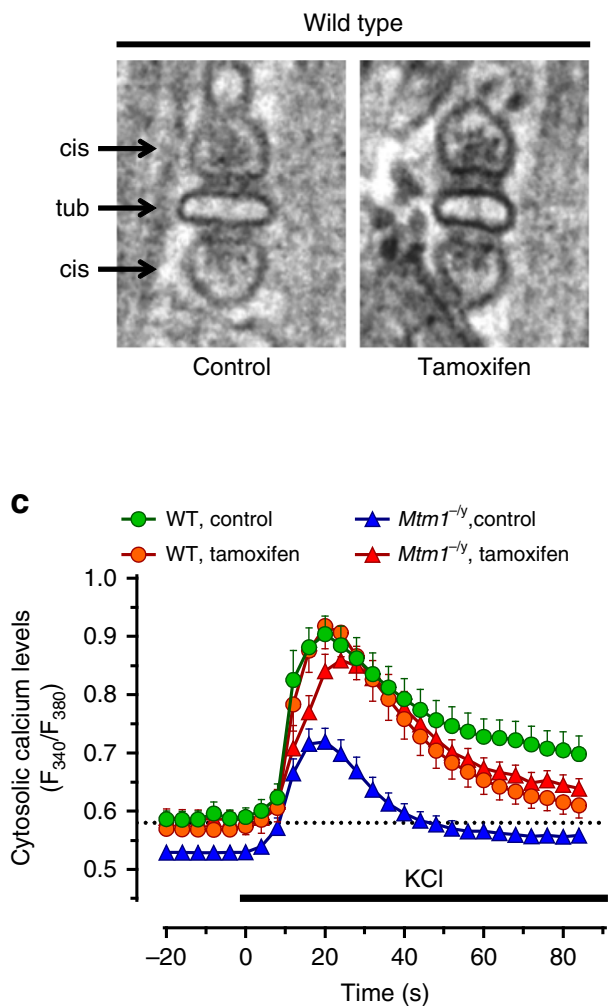

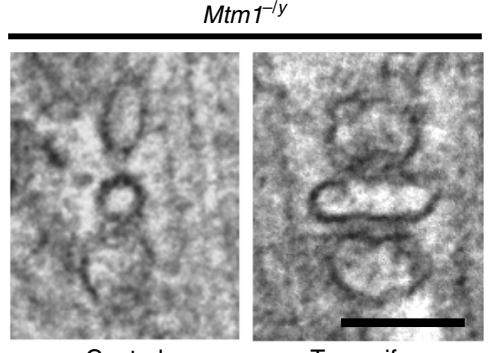

Control

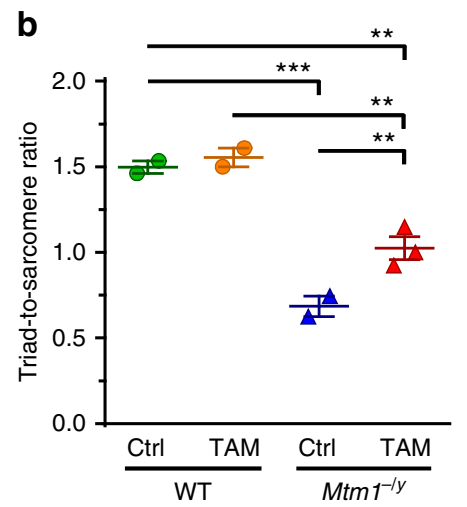

e

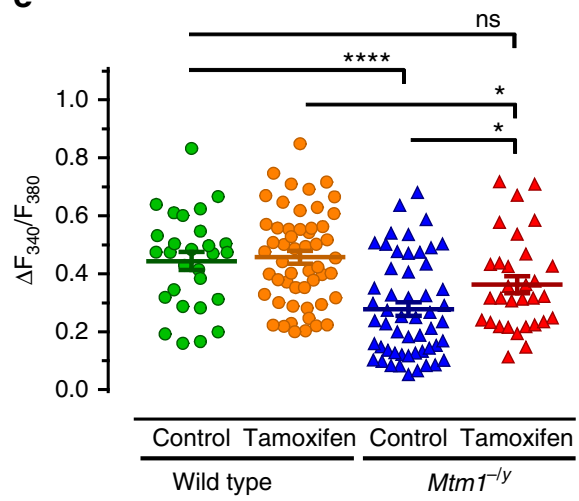

$\mathbf{f}$

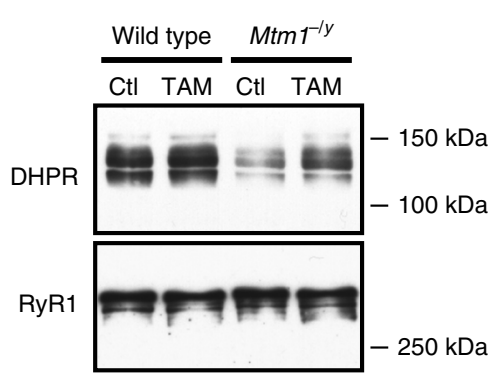

d

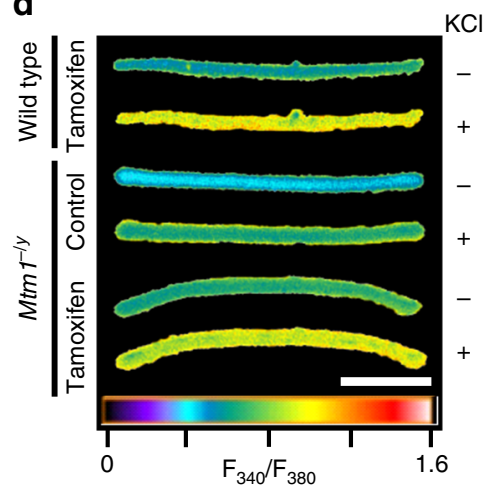

b

Tamoxifen
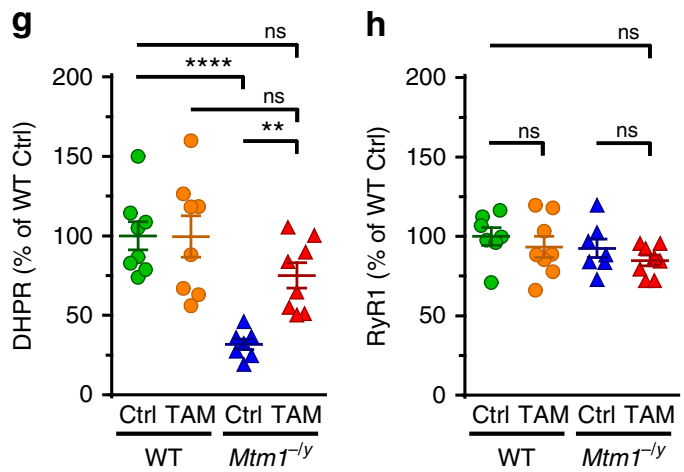

Fig. 5 Tamoxifen improves triad density and excitation-contraction coupling, Triads and excitation-contraction coupling were examined in 42 days old (D42) mice either untreated (control; Ctrl) or treated with tamoxifen (TAM; $30 \mathrm{mg} \mathrm{kg}^{-1}$ in diet). a Triads-specialized membrane structures made of a ttubule (tub) flanked by terminal cisternae (cis) arising from the sarcoplasmic reticulum and controlling $\mathrm{Ca}^{2+}$ release-were visualized by transmission electron microscopy (TEM) in the tibialis anterior (TA) of mice at D42. Note the abnormal shape of the remaining triads in the $M t m 1^{-/ y}$ mouse and recovery with tamoxifen treatment. The bar represents $100 \mathrm{~nm}$. b The number of well-positioned triads per sarcomere unit was determined from TEM pictures in TA from wild type (WT) and Mtm1 $1^{-/ y}$ mice at D42. TAM partly rescued the much decreased triad density in Mtm ${ }^{-/ y}$ mice. Mean \pm s.e.m. of $n$ =2-3 mice. c-e Excitation-contraction coupling assessed via live imaging of $\mathrm{Ca}^{2+}$ fluxes induced by $\mathrm{KCl}$ depolarization in single FDB myofibers (see Online Methods for details). c Average traces of the responses of FDB fibers to $\mathrm{KCl}$, an experimental setting that mimics muscle depolarization ( $n=17-29$ fibers). d Representative images (pseudo-colored) illustrating cytosolic $\mathrm{Ca}^{2+}$ levels in $\mathrm{FDB}$ fibers at baseline (-; before $\mathrm{KCl}$ pulse) and at the peak of $\mathrm{KCl}$-induced response (+). The bar represents $100 \mu \mathrm{m}$. e Quantification of cytosolic $\mathrm{Ca}^{2+}$ at the peak response. TAM enhanced $\mathrm{Mtm}^{-/ \mathrm{y}} \mathrm{cytosolic} \mathrm{Ca}{ }^{2+}$ to levels found in WT. Data shown in e represent, from left to right, the mean \pm s.e.m. of $n=29,53,53$, and 31 fibers. $\mathbf{f}$ Representative western blots of DHPR and RyR1. $\mathbf{g}$, h Quantification of DHPR and RyR1, respectively. Mean \pm s.e.m. of $n=7$ muscles. ${ }^{\star} P \leq 0.05 ;{ }^{\star \star} P \leq 0.01 ;{ }^{\star \star \star} P \leq 0.001 ;{ }^{\star \star \star \star} P \leq 0.0001 ;$ ns non-significant. One-way ANOVA followed by Fisher's LSD post-test

breeding wild type males with $M t m 1^{+/-}$heterozygous females. The $M t m 1^{+/ y}$ (wild type) and $M t m 1^{-/ y}$ (mutant) males in the progeny were used in experiments. Mice were housed on wood granule bedding in either Eurostandard type II or type III polycarbonate cages equipped with filter tops (Indulab AG, Gams, Switzerland). They were kept on a 12 -h dark/12-h light cycle, under constant temperature $(22 \pm$ $1{ }^{\circ} \mathrm{C}$ ) and humidity (50-60\%). Mice were allowed unlimited access to food and water throughout the study. Environment enrichment consisted in a Mouse House (Indulab AG), shredded paper, and cylinders of pure cellulose (Cocoon, Datesand, Manchester, UK).
Genotyping. Toe biopsies were taken at 7-10 days post-natal for combined genotyping and permanent marking of individuals. DNA was prepared using DirectPCR Lysis Reagent/Mouse Tail (AxonLab AG, Le Mont-sur-Lausanne, Switzerland) according to the manufacturer's instructions. The final lysate was diluted fivefold in molecular biology grade water. One $\mu \mathrm{L}$ of DNA was used for PCR amplification using ready to load 2x GoTaq ${ }^{\circledR}$ Master Mix (Promega, Dübendorf, Switzerland). The following primers (Microsynth AG, Balgach, Switzerland) were added: MTM1 Forward 1 (5'-AGACAGTGATGCAC AGAGAGGAG- $3^{\prime}$ ), binding upstream of exon 4 (used at $0.25 \mu \mathrm{M}$ ); MTM1 
a

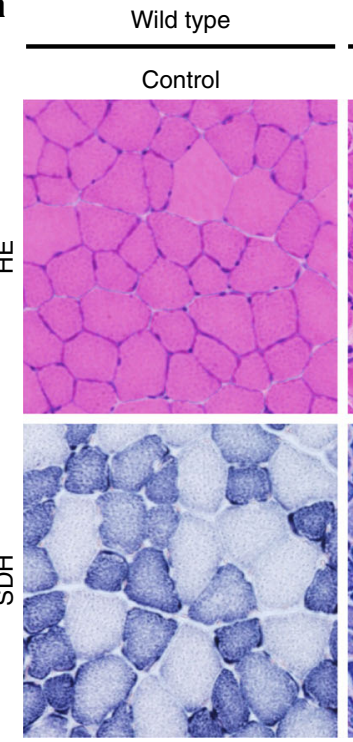

D42

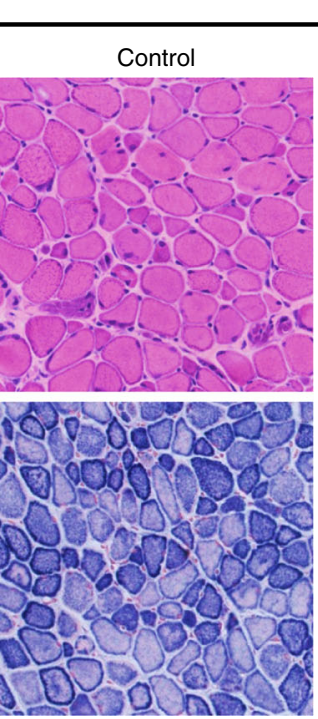

D42

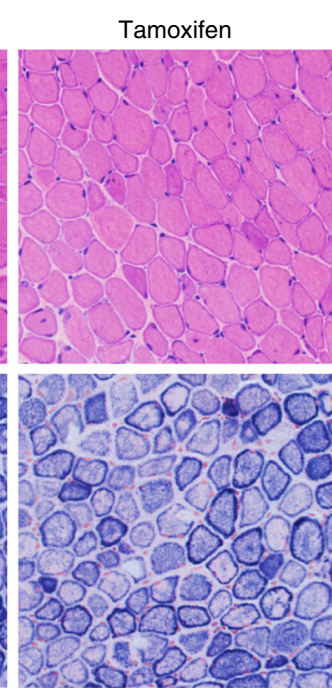

D42
$M \mathrm{Mm}^{-/ y}$

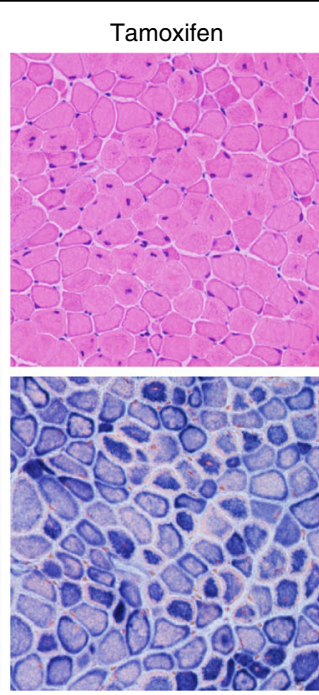

D84

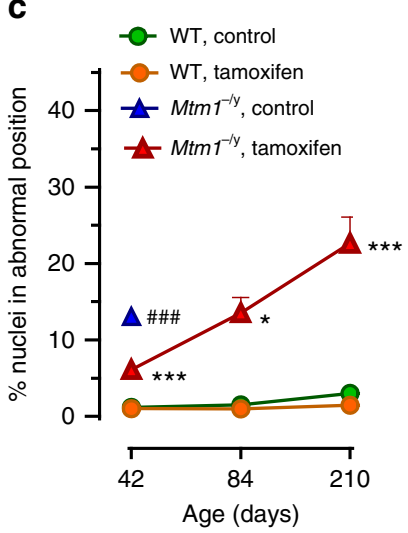

Age (days)

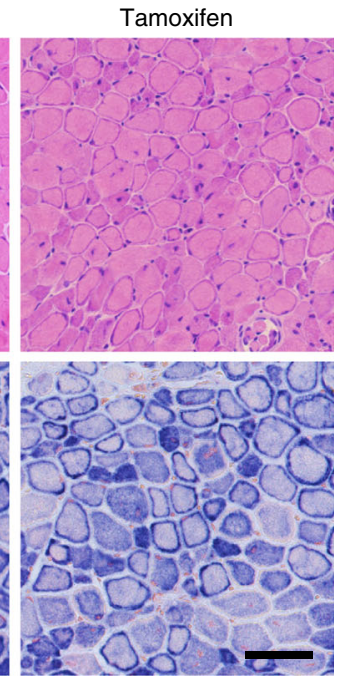

D210

d

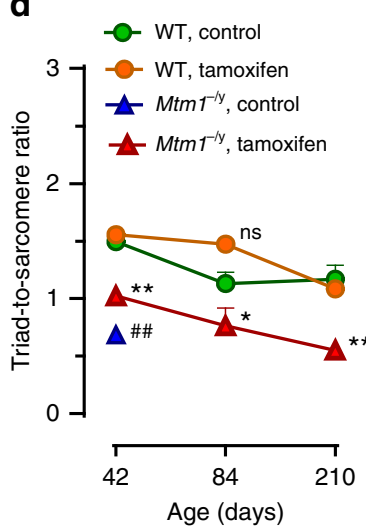

b

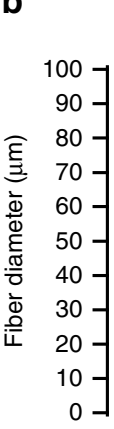
$\stackrel{* \star \star * *}{\stackrel{* * * * *}{* * *}}$ Control
Tamoxifen Wild type

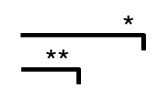

$\varnothing$

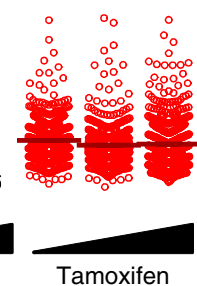

$M t m 1^{-l y}$
Tamoxifen

Fig. 6 Myopathic features after long-term tamoxifen treatment, Muscle structure and triads were examined by histology and transmission electron microscopy, respectively, in wild type (WT) and $M t m 1^{-/ y}$ mice during long-term treatment with tamoxifen ( $30 \mathrm{mg} \mathrm{kg}^{-1}$ in diet). a Representative pictures of tibialis anterior (TA) sections from untreated WT and Mtm1 $1^{-/ y}$ mice at D42, and tamoxifen-treated Mtm1 ${ }^{-/ y}$ mice at D42, D84, and D210 stained with hematoxylin-eosin (HE) or for succinate dehydrogenase (SDH) activity. Muscle structure of $M t m 1^{-/ y}$ mice was stable over the duration of tamoxifen treatment. The bar represents $50 \mu \mathrm{m}$. b Scatter plots showing the distribution of TA myofiber diameter in mice at D42, D84, and D210 (black triangles illustrate increasing age). Fiber size remained almost constant from D42 to D210. $N=600$ myofibers per group. $\mathbf{c}$ The percentage of abnormally positioned nuclei (either internally or centrally located) in TA myofibres of mice at D42, D84, and D210 were counted from hematoxylin-eosin-stained sections. The density of abnormally positioned nuclei increased significantly with ageing. Mean \pm s.e.m. of $n=2-5$ TA per group. $\mathbf{d}$ The number of well-positioned triads relative to sarcomere number, determined from TEM pictures in TA from wild type and Mtm1-/y mice, showed no significant change from D42 to D84. Black triangles illustrate increasing age (42-84 and 210 days). Mean \pm s.e.m. of $n=2-3$ TA per group. ${ }^{\star} P \leq 0.05 ;{ }^{\star \star} P \leq 0.01 ;{ }^{* \star \star} P \leq 0.001 ;{ }^{\star \star \star \star \star} P \leq 0.0001$. $\# \# \leq 0.01 ; \mathrm{Mtm}^{-/ \mathrm{y}}$ control vs tamoxifen at D42. ns non-significant; $\varnothing$ no surviving mice. One-way ANOVA followed by Fisher's LSD post-test

Forward 2 (5'-AATGGCCCCATTAAGGGAAG-3'), binding within exon 4 (used at $0.50 \mu \mathrm{M})$; MTM1 Reverse (5'-GTGTGCATGTTTGGACCATGG-3'), binding downstream of exon 4 (used at $0.50 \mu \mathrm{M})$. The PCR reaction ( $10 \mu \mathrm{L}$ final volume) was run on a Biometra Tpersonal thermocycler (AxonLab) as follows: initial denaturation at $95^{\circ} \mathrm{C}$ for $5 \mathrm{~min}$; amplification: denaturation at $95^{\circ} \mathrm{C}$ for $20 \mathrm{~s}$, annealing at $55^{\circ} \mathrm{C}$ for $30 \mathrm{~s}$, elongation at $72^{\circ} \mathrm{C}$ for $60 \mathrm{~s}, 35$ cycles; final elongation at $72^{\circ} \mathrm{C}$ for $5 \mathrm{~min}$; holding temperature, $10^{\circ} \mathrm{C}$. PCR products were analyzed on $1 \%$ agarose gels containing SYBR Safe DNA Gel Stain (Thermo Fisher Scientific AG, Reinach, Switzerland), at a 1:20,000 dilution. The mutant allele was identified as a band of $165 \mathrm{bp}$ and the wild type one as a band of $437 \mathrm{bp}$ (a band of $920 \mathrm{bp}$ arising from the primers binding outside exon 4 was expected but not efficiently amplified).

Groups and treatments. $M t m 1^{+1-}$ carrier females are clinically healthy. Only male mice were used in experiments. Male pups were weaned on post-natal day 23 \pm 1 , at which time treatments were initiated. Randomization was not feasible because small cohorts were generally produced and each group had to comprise enough wild type mice to foster the mutants. Sub-groups of mice, usually made of 2-4 wild type and 1-4 Mtm $1^{-/ y}$ mice, were given pellets of diet supplemented with different amounts of tamoxifen citrate (Haohua Industry Co., Jinan City, China) to achieve to 3,10, and $30 \mathrm{mg}$ of tamoxifen free base per $\mathrm{kg}$ of chow. The modified chow was prepared by Cargill, Provimi-Kliba AG (Kaiseraugst, Switzerland).

Specific care was systematically implemented in order to restrict suffering of the fragile $M t m 1^{-/ y}$ mice. This included food pellets placed inside the cages, bottles equipped with long nozzles, bedding granules to raise the ground and facilitate access to drinking water, nestling and enrichment materials, and non-experimental chaperone mice in case not enough wild type littermates were generated along with the $M t m 1^{-/ y}$ mice.

For survival analyses, mice were followed up to 200-210 days of age. For the other experiments, treatments were maintained until post-natal day $42( \pm 1)$ days (D42, young mice), at which time around $60 \%$ of the untreated $\mathrm{Mtm}^{-/ \mathrm{y}}$ mice were still alive; $84( \pm 2)$ days (D84, adult mice), at which time none of the untreated $M t m 1^{-1 y}$ mice survived; and $210( \pm 3)$ days (D210, old mice). 
Longitudinal monitoring of disease progression. Body weight, food consumption, and clinical score were recorded three times per week on each sub-group of mice. Food consumption (in $\mathrm{g}$ diet per $\mathrm{g}$ body weight per day) was calculated from the amount of diet (in g) consumed by each cage (average total body weight in g) during periods of 2-3 consecutive days. Disease progression was assessed three times per week using a clinical grade scale adapted from that described earlier ${ }^{38}$. In brief, the mice were given a score of 1 (normal function of hind limbs), 2 (difficulty in spreading toes), 3 (evident weakness in legs), 4 (paralysis of one hind limb), or 5 (both hind limbs are paralyzed). Moribund mice were killed, usually soon after reaching stage 5 .

Mice were subjected once a week to a horizontal grid-hanging test that measured their ability to sustain their own body weight against gravity. In brief, a mouse was placed on the center of a wire grid (dimension $35 \times 50 \mathrm{~cm}$, made of $1 \mathrm{~mm}$-diameter metal wire and forming a $1.26 \mathrm{~cm}$ square mesh). The grid was gently inverted upside-down and maintained approximately $50 \mathrm{~cm}$ above a thick layer of soft bedding material to avoid causing damage to the animals in case of fall. The time until which the mouse fell from the grid was recorded. In the event of a fall, the timer was stopped, the grid put back to its original position, the mouse promptly returned to the grid and the test was continued. The test was ended when the final time score (set at $60 \mathrm{~s}$ ) was reached or when the mouse was not able to hold the grid for more than $10 \mathrm{~s}$ between consecutive falls.

\section{Electrically evoked contractions of the triceps surae. Muscle mechanical} properties of the triceps surae (hereafter referred to as "triceps") were recorded in situ in deeply sedated, freely breathing mice without disturbing triceps innervation and blood supply. The triceps is a large muscle group that makes up most of the calf volume and comprises the gastrocnemius, the plantaris and the soleus muscles. Its balanced composition of slow- and fast-contracting fibers makes it similar to most human leg muscles, hence its value for physiology, experimental pharmacology and evaluating therapeutics. In an isometric setting such as the one we used, the muscle is set to a fixed length. Electrical stimulation causes attempts of the muscle to contract; the mechanical tension ("force") exerted on its extremities is measured via a force transducer. Meaningful parameters are determined from two extreme mechanical responses that are phasic and tetanic contractions. A phasic (also known as twitch) contraction consists in an elementary muscle contraction, comprising a rising phase followed by full muscle relaxation (return to baseline), from which kinetics of contraction and relaxation can be calculated. The phasic force is measured as the amplitude of the response (see Fig. 2a, b in the main text). On the other end of the spectrum, stimulating the muscle at high frequency generates a tetanic contraction, which is the maximum force that the muscle can produce (Fig. 2d, e).

At the end of the treatment period, mice were briefly sedated by inhalation of isoflurane before i.p. injection of a mixture of urethane-diazepam-buprenorphine $\left(1.5 \mathrm{~g} \mathrm{~kg}^{-1}, 5 \mathrm{mg} \mathrm{kg}-1\right.$, and $100 \mathrm{\mu g} \mathrm{kg}^{-1}$, respectively), ensuring deep anesthesia and adequate analgesia for over $2 \mathrm{~h}$. Mechanical responses of the right triceps to electrical stimulations were recorded isometrically using a custom-made device as previously described ${ }^{36,70-74}$. In brief, the knee joint was firmly immobilized, and the Achilles tendon was linked to a force transducer coupled to a LabView interface (National Instruments, Austin, TX). Two thin steel electrodes were inserted into the triceps for delivering 0.5 -ms pulses of controlled intensity and frequency. After manual settings of optimal muscle length and optimal current intensity, 5-6 phasic responses to single stimuli were recorded at a sampling rate of $3 \mathrm{kHz}$ to determine absolute peak twitch tension, time to peak twitch tension and time for half relaxation from peak twitch tension. Then, force-frequency curves were constructed at a sampling rate of $1 \mathrm{kHz}$ : the triceps was subjected to $200-\mathrm{ms}$ trains of stimuli at increasing frequencies ( 10 to $120 \mathrm{~Hz}$ by increments of $10 \mathrm{~Hz}$, then 150 and $200 \mathrm{~Hz}$ ) delivered at 30 -s intervals. The maximum response was taken as the absolute tetanic tension. Absolute phasic and absolute tetanic tensions were converted into specific tensions (in $\mathrm{mN} \mathrm{mm}^{-2}$ of muscle section) after normalization for the muscle cross-sectional area. The cross-sectional area (in $\mathrm{mm}^{2}$ ) was determined by dividing the triceps mass (in $\mathrm{mg}$ ) by the product of the optimal muscle length (in $\mathrm{mm}$ ) and the density of mammalian skeletal muscle $\left(1.06 \mathrm{mg} \mathrm{mm}^{-3}\right)$.

Blood and tissue sampling. Immediately after isometric force recordings, mice were thoracotomized, heparin (LKT labs, Enzo Life Sciences, Lausen, Switzerland) (MW 4-6 kDa; $30 \mu \mathrm{L} ; 30 \mathrm{U} \mathrm{mL}^{-1}$ ) was injected into the heart, and the descending aorta was cut. Total blood was collected in a $1.5-\mathrm{mL}$ microcentrifuge tube, and centrifuged $\left(10 \mathrm{~min}, 4^{\circ} \mathrm{C}, 10,000 \times \mathrm{g}\right)$. Plasma was snap-frozen in liquid nitrogen before being stored at $-80^{\circ} \mathrm{C}$. Volumes of total blood and plasma were recorded. Selected muscles were quickly dissected and weighed (Supplementary Table 2 and 3). Tibialis anterior (TA) were processed for histology and transmission electron microscopy. The gastrocnemius were saved for RT-PCR and western-blot analyses. The remaining leg muscles were collected and pooled. That muscle bulk was saved for determining levels of tamoxifen and its metabolites. In some instances, flexor digitorum brevis (FDB) muscles were used for live imaging of calcium fluxes in single intact fibers.
Histological analysis of skeletal muscle. TA muscles were embedded in tragacanth gum (Sigma-Aldrich) $(5 \% \mathrm{w} / \mathrm{v}$ in water), frozen in liquid nitrogen-cooled isopentane and stored at $-80^{\circ} \mathrm{C}$ until further processing. Air-dried $10 \mu \mathrm{m}$-thick transverse sections were fixed with 4\% PFA and stained with hematoxylin and eosin (HE) or for succinate dehydrogenase (SDH) activity using conventional procedures ${ }^{16,17}$. Image acquisition was performed with a NanoZoomer 2.0-HT slide scanner equipped with the fluorescence module L11600-21 (Hamamatsu Photonics). Myofiber cross-sectional area (CSA) was analyzed using FIJ image analysis software (ImageJ 1.51s version, available at http://imagej.net/) from fluorescence pictures taken from HE-stained TA sections. CSA $\left(\mu \mathrm{m}^{2}\right)$ was determined from at least 300 fibers per muscle. The percentage of myofibers with abnormally positioned nuclei (either centralized or internalized) was calculated from at least 300 fibers per TA using the cell counter plugin in FIJI analysis software. The total number of myofibers in each TA was also counted using FIJI.

Transmission electron microscopy. The proximal half of TA muscles was cut into small pieces that were fixed with $2.5 \%$ glutaraldehyde, $2.5 \%$ paraformaldehyde and $50 \mathrm{mM} \mathrm{CaCl}_{2}$ in $0.1 \mathrm{M}$ cacodylate buffer (pH 7.2). Samples were post-fixed with $2 \%$ $\mathrm{OsO}_{4}, 0.8 \% \mathrm{~K}_{3} \mathrm{Fe}(\mathrm{CN})_{6}$ in $0.1 \mathrm{M}$ cacodylate buffer $(\mathrm{pH} 7.4)$ for $2 \mathrm{~h}$ a t $4^{\circ} \mathrm{C}$ and incubated with $5 \%$ uranyl acetate for $2 \mathrm{~h}$ at $4{ }^{\circ} \mathrm{C}$. Muscles were dehydrated in a graded series of ethanol and embedded in epon resin. Thin $(70-\mathrm{nm})$ sections were stained with uranyl acetate and lead citrate and examined by transmission electron microscope. The number of triads per sarcomere (around 40 per mouse) was quantified from electron micrographs using the Metamorph 3 software $e^{15,75}$. The ratio of triads/sarcomere was calculated by dividing number of triads clearly identified and correctly localized by the total number of sarcomeres present in the image $e^{15,76}$.

Analysis of mRNA expression by quantitative RT-PCR. The left gastrocnemius muscle (non-exposed to the isometric contraction protocol) was snap-frozen in liquid nitrogen and stored at $-80^{\circ} \mathrm{C}$ until processed for quantitative PCR (qPCR) essentially as described ${ }^{36}$. The muscles were ground to a fine powder in mortars cooled in liquid nitrogen. RNA were extracted from approximately $10 \mathrm{mg}$ of muscle powder using RNeasy Fibrous Tissue mini kit (Qiagen, Hombrechtikon, Switzerland). Tissue disruption was enhanced by sonicating three times for $3 \mathrm{~s}$ on ice. Then $100 \mathrm{ng}$ of total RNA were reverse-transcribed with Super-Script II Reverse Transcriptase (Invitrogen). The resulting cDNA was subjected to quantitative PCR (qPCR) amplification using PowerUp SYBR ${ }^{\mathrm{TM}}$ Green Master mix (Applied Biosystems, Thermo Fisher Scientific) according to the manufacturer's instructions. Briefly, $10 \mu \mathrm{L}$ reaction mixtures were prepared containing $1 \mu \mathrm{L}$ of cDNA (equivalent to $2.5 \mathrm{ng}$ of initial RNA), forward and reverse primers (500 nM each) for Bin1 (pan isoforms), Bin1 (isoform 8), Dnm2, Esr1, Esr2, Pik3c2b or Gapdh (used as the housekeeping gene) (Supplementary Table 4), the kit Master mix and RNAse-free water. They were subjected to PCR amplification using a StepOnePlus Real-Time PCR System thermocycler (Thermo Fisher Scientific) under the following conditions: initial denaturation at $95^{\circ} \mathrm{C}$ for $60 \mathrm{~s}$; amplification: denaturation at $95^{\circ} \mathrm{C}$ for $20 \mathrm{~s}$, annealing at $55^{\circ} \mathrm{C}$ for $30 \mathrm{~s}$, elongation at $72^{\circ} \mathrm{C}$ for $60 \mathrm{~s}, 40 \mathrm{cycles}$; final elongation at $72^{\circ} \mathrm{C}$ for $5 \mathrm{~min}$. The expression level of each transcript was expressed relative to that of Gapdh using the $2^{-\Delta \Delta C t}$ method $^{36}$.

Semi-quantitative western-blots. Gastrocnemius muscles were pulverized on liquid nitrogen and extracts were prepared as previously described ${ }^{36,73,74}$. The final protein concentration was adjusted to $3 \mathrm{mg} \mathrm{mL}^{-1}$ with reducing Laemmli buffer. Muscle extracts (30 to $60 \mu \mathrm{g}$ per lane) were resolved by SDS-PAGE, and proteins were transferred onto nitrocellulose membranes using standard procedures. Equal loading and transfer efficiency were verified with Ponceau Red staining. Membranes were blocked for $1 \mathrm{~h}$ in TBST $(20 \mathrm{mM}$ Tris-base, $150 \mathrm{mM} \mathrm{NaCl}, 0.05 \%$ Tween-20, pH 7.5) containing 5\% non-fat dry milk and incubated overnight at $4{ }^{\circ} \mathrm{C}$ with one of the primary antibodies listed in Supplementary Table 5.

After extensive wash in TBST, the membranes were incubated with a HRPconjugated secondary reagent (Donkey anti-Rabbit (Amersham, GE Healthcare Life Sciences, Thermo Fisher Scientific), Goat anti-mouse (Bio-Rad), or kappa light chain binding protein (Santa Cruz Biotechnologies)) as required before signal detection on X-ray Fuji films (Thermo Fisher Scientific) using an enhanced chemiluminescence kit (ECL Prime Western Blotting Detection Reagent) (Amersham). The X-ray films were scanned at high resolution (720 dpi) on an Epson Perfection V750 PRO scanner using settings for transparent documents in order to avoid signal saturation and augment the linear range of the signals. Bands were quantified using ImageJ (version 1.48; freely available at http://imagej.nih.gov/ij). Specific care allowing semi-quantitative analysis has been described in details elsewhere ${ }^{36,74}$. Briefly, in order to allow intra-gel and inter-gel comparison and semi-quantitative analysis of the signals, the following procedure was applied: (i) for each muscle protein to be quantified, 4 gels were run simultaneously (PerfectBlue ${ }^{\text {tw }}$ Dual Gel System Twin ExW S, Peqlab) and processed in parallel, (ii) the samples (6-8 muscles per group) were loaded as quadruplets, each consisting of extracts from non-treated and TAM-treated WT and non-treated and TAM-treated Mtm1-null mice (iii) each quadruplet was flanked by a standard extract (Std), consisting of a mixture of all muscle extracts, (iv) the portions of the gels that contained the protein of interest were transferred onto a single nitrocellulose membrane, ensuring that all samples and flanking standard extracts were 
simultaneously exposed to the blocking solution, primary antibodies, secondary HRPconjugated secondary antibody, ECL reagent, and X-ray film. Signals were normalized to GAPDH and the resulting values were normalized to that of the flanking standard samples. The standard sample being a mixture of all samples to be compared the ones with the others, this ensured that its signal has an average, non-saturating, intensity. Finally, the values were expressed as the percentage of the WT control group.

Larger views of the blots for which portions are shown in Figs. 4 and 5 are presented in Supplementary Figure 6.

Culture of isolated flexor digitorum brevis (FDB) fibers. Hind feet were dissected and immersed in transfer buffer (Hank's buffered salt solution supplemented with $10 \mathrm{mM}$ HEPES, pH 7.4). FDB muscles were dissected under a binocular microscope, transferred to warmed $\left(37^{\circ} \mathrm{C}\right)$ maintenance medium (Dulbecco's Modified Eagle's Medium (DMEM) containing 10\% fetal bovine serum and $10 \mu \mathrm{g} \mathrm{mL}^{-1}$ ciprofloxacin (Sigma)), and $1 \mathrm{mg} \mathrm{mL}^{-1}$ collagenase type IA (Sigma) was finally added ${ }^{77}$. The full process took no more than $1 \mathrm{~h}$. The mixture was maintained for $60 \mathrm{~min}$ at $37^{\circ} \mathrm{C}$ with frequent shaking in a humidified incubator gassed with air containing $5 \% \mathrm{CO}_{2}$. Then, muscles were carefully rinsed twice in warmed maintenance medium to remove enzyme excess and individual fibers were released by triturating gently with fire-polished Pasteur pipettes of decreasing opening widths. Throughout that process, aliquots of maintenance medium containing single fibers were transferred onto clean $18 \mathrm{~mm}$-diameter glass coverslips coated with $15 \mu \mathrm{g} \mathrm{cm}^{-2}$ Matrigel (BD biosciences) in 12-well tissue culture plates. $\mathrm{Ca}^{2+}$ measurement was performed the next day.

Live imaging of cytosolic calcium in FDB fibers. $\mathrm{Ca}^{2+}$ levels in the cytosol of FDB fibers was monitored via fluorescence microscopy using the calcium-sensitive probe Fura-2 essentially as described previously ${ }^{77}$. Fura-2-AM (Molecular Probes, Invitrogen, Thermo Fisher Scientific), the acetoxymethyl ester of Fura-2, was used to facilitate loading into fibers and the subsequent de-esterification and intracellular trapping. The Fura-2-AM stock solution was prepared extemporaneously by mixing Fura-2-AM (5 mM) with an equal volume of Pluronic acid F-127 (20\% w/v solution, Molecular probes). Fibers were washed twice with physiological salt solution containing $\mathrm{Ca}^{2+}\left(\mathrm{PSS}^{+}\right.$; composition in mM: HEPES 5, KCl 5, $\mathrm{MgCl}_{2} \cdot 6 \mathrm{H}_{2} \mathrm{O} 1, \mathrm{NaCl} 145, \mathrm{CaCl}_{2} \cdot 2 \mathrm{H}_{2} \mathrm{O} 1.7$, glucose 10) before incubation in the loading solution (PSS ${ }^{+}$containing $2 \mu \mathrm{L} \mathrm{mL}^{-1}$ of the Fura-2-AM/Pluronic acid working solution; final Fura- 2 concentration $5 \mu \mathrm{M}$ ). Loading was done at $37^{\circ} \mathrm{C}$ for $45 \mathrm{~min}$ after which the cells were washed twice with PSS ${ }^{+}$and Fura-2-AM allowed to de-esterify at room temperature for another $20 \mathrm{~min}$. Loading and deesterification buffers were supplemented with $100 \mu \mathrm{M} \mathrm{N}$-benzyl-p-toluenesulfonamide (BTS) (Medchem Express, Lucerna-Chem, Luzern, Switzerland) to prevent fiber contraction and detachment. All loading steps were done in the dark. Coverslips holding fibers were placed in a perfusion chamber that was continuously perfused at $1.5 \mathrm{~mL} \mathrm{~min}^{-1}$ using a peristaltic pump. The imaging setup consisted of a high speed monochromator (Visitron Systems, Puchheim, Germany) allowing selection of alternating excitation wavelengths and an ORCA-Flash4.0LT Digital sCMOS camera mounted on an Axiovert 200 microscope (Carl Zeiss AG, Feldbach, Switzerland) equipped with objectives for high resolution fluorescence imaging. The experiments were done ratiometrically with alternating excitation wavelengths of 340 and $380 \mathrm{~nm}$ and emission at wavelengths above $510 \mathrm{~nm}$ using a long pass filter. Images were acquired every $2 \mathrm{~s}$. After recording baseline calcium, fibers were perfused with a modified PSS ${ }^{+}$containing high concentration of $\mathrm{KCl}(125 \mathrm{mM})$ and reduced concentration of $\mathrm{NaCl}(10 \mathrm{mM}) . \mathrm{Ca}^{2+}$ increment during depolarization was calculated as the difference between resting $\mathrm{Ca}^{2+}$ levels and the maximum levels of $\mathrm{Ca}^{2+}$ reached during depolarization. Data acquisition and analysis was performed using VisiView software version 3.3.0.3 (Visitron Systems).

Data analysis and statistics. Survival curves were analyzed using the Log-rank (Mantel-Cox) test. All other comparisons were assessed by ANOVA followed by Fisher's least significance difference (LSD) test. The overall changes in body weights, grid test scores and clinical grades over time were assessed from the areas under the curves calculated from every mouse.

\section{Data availability}

The authors declare that all data supporting the findings of this study are available within the paper and its supplementary information files. A reporting summary for this Article is available as a Supplementary Information file.

Received: 10 September 2018 Accepted: 12 October 2018

Published online: 19 November 2018

\section{References}

1. Jungbluth, H. \& Gautel, M. Pathogenic mechanisms in centronuclear myopathies. Front. Aging Neurosci. 6, 339 (2014).
2. Lawlor, M. W. et al. Skeletal muscle pathology in X-Linked myotubular myopathy: review with cross-species comparisons. J. Neuropathol. Exp. Neurol. 75, 102-110 (2016).

3. Romero, N. B. Centronuclear myopathies: a widening concept. Neuromuscul. Disord. 20, 223-228 (2010).

4. Barth, P. G. \& Dubowitz, V. X-linked myotubular myopathy--a long-term follow-up study. Eur. J. Paediatr. Neurol. 2, 49-56 (1998).

5. Buj-Bello, A., Biancalana, V., Moutou, C., Laporte, J. \& Mandel, J.-L. Identification of novel mutations in the MTM1 gene causing severe and mild forms of X-linked myotubular myopathy. Hum. Mut. 14, 320-325 (1999).

6. Biancalana, V. et al. Affected female carriers of MTM1 mutations display a wide spectrum of clinical and pathological involvement: delineating diagnostic clues. Acta Neuropathol. 134, 889-904 (2017).

7. Blondeau, F. et al. Myotubularin, a phosphatase deficient in myotubular myopathy, acts on phosphatidylinositol 3-kinase and phosphatidylinositol 3phosphate pathway. Hum. Mol. Genet. 9, 2223-2229 (2000).

8. Tronchère, H. et al. Production of phosphatidylinositol 5-phosphate by the phosphoinositide 3-phosphatase myotubularin in mammalian cells. J. Biol. Chem. 279, 7304-7312 (2004)

9. Taylor, G. S., Maehama, T. \& Dixon, J. E. Myotubularin, a protein tyrosine phosphatase mutated in myotubular myopathy, dephosphorylates the lipid second messenger, phosphatidylinositol 3-phosphate. Proc. Natl Acad. Sci. USA 97, 8910-8915 (2000).

10. Bachmann, C. et al. Cellular, biochemical and molecular changes in muscles from patients with X-linked myotubular myopathy due to MTM1 mutations. Hum. Mol. Genet. 26, 320-332 (2017).

11. Elverman, M. et al. Long-term effects of systemic gene therapy in a canine model of myotubular myopathy. Muscle Nerve 56, 943-953 (2017).

12. Mack, D. L. et al. Systemic AAV8-mediated gene therapy drives wholebody correction of myotubular myopathy in dogs. Mol. Ther. 25, 839-854 (2017).

13. Childers, M. K. et al. Gene therapy prolongs survival and restores function in murine and canine models of myotubular myopathy. Sci. Transl. Med. 6, 220ra210 (2014).

14. Buj-Bello, A. et al. AAV-mediated intramuscular delivery of myotubularin corrects the myotubular myopathy phenotype in targeted murine muscle and suggests a function in plasma membrane homeostasis. Hum. Mol. Genet. 17, 2132-2143 (2008)

15. Cowling, B. S. et al. Reducing dynamin 2 expression rescues X-linked centronuclear myopathy. J. Clin. Invest. 124, 1350-1363 (2014).

16. Tasfaout, H. et al. Antisense oligonucleotide-mediated Dnm2 knockdown prevents and reverts myotubular myopathy in mice. Nat. Commun. 8, 15661 (2017).

17. Cowling, B. S. et al. Amphiphysin (BIN1) negatively regulates dynamin 2 for normal muscle maturation. J. Clin. Invest. 127, 4477-4487 (2017).

18. Ismail, H. M., Dorchies, O. M. \& Scapozza, L. The potential and benefits of repurposing existing drugs to treat rare muscular dystrophies. Exp. Opin. Orphan Drugs 6, 259-271 (2018).

19. Hayes, T. G. Pharmacologic treatment of male breast cancer. Expert. Opin. Pharmacother. 10, 2499-2510 (2009).

20. Shagufta, AhmadI. Tamoxifen a pioneering drug: an update on the therapeutic potential of tamoxifen derivatives. Eur. J. Med. Chem. 143, 515-531 (2018).

21. Peddi, P. F. Hormone receptor positive breast cancer: state of the art. Curr. Opin. Obstet. Gynecol. 30, 51-54 (2018).

22. Moein, M. R., Tabibnejad, N. \& Ghasemzadeh, J. Beneficial effect of tamoxifen on sperm recovery in infertile men with nonobstructive azoospermia. Andrologia 44, 194-198 (2012).

23. Chua, M. E. et al. Revisiting oestrogen antagonists (clomiphene or tamoxifen) as medical empiric therapy for idiopathic male infertility: a meta-analysis. Andrology 1, 749-757 (2013).

24. Patel, D. P., Chandrapal, J. C. \& Hotaling, J. M. Hormone-based treatments in subfertile males. Curr. Urol. Rep. 17, 56 (2016).

25. Kunath, F., Keck, B., Antes, G., Wullich, B. \& Meerpohl, J. Tamoxifen for the management of breast events induced by non-steroidal antiandrogens in patients with prostate cancer: a systematic review. BMC Med. 10, 96 (2012).

26. Payne, W. G. et al. Down-regulating causes of fibrosis with tamoxifen: a possible cellular/molecular approach to treat rhinophyma. Ann. Plast. Surg. 56, 301-305 (2006).

27. van Bommel, E. F., Hendriksz, T. R., Huiskes, A. W. \& Zeegers, A. G. Brief communication: tamoxifen therapy for nonmalignant retroperitoneal fibrosis. Ann. Intern. Med. 144, 101-106 (2006).

28. Walter, A. W. et al. Tamoxifen and carboplatin for children with low-grade gliomas: a pilot study at St. Jude Children's Research Hospital. J. Pediatr. Hematol. Oncol. 22, 247-251 (2000).

29. Lawrence, S. E., Arnold Faught, K., Vethamuthu, J. \& Lawson, M. L. Beneficial effects of raloxifene and tamoxifen in the treatment of pubertal gynecomastia. J. Pediatr. 145, 71-76 (2004). 
30. Skapek, S. X. et al. Safety and efficacy of high-dose tamoxifen and sulindac for desmoid tumor in children: results of a Children's Oncology Group (COG) Phase II Study. Pediatr. Blood. Cancer 60, 1108-1112 (2013).

31. Kreher, N. C., Eugster, E. A. \& Shankar, R. R. The use of tamoxifen to improve height potential in short pubertal boys. Pediatrics 116, 1513-1515 (2005).

32. Lapid O., van Wingerden J. J., \& Perlemuter L. Tamoxifen therapy for the management of pubertal gynecomastia: a systematic review. J. Pediatr. Endocrinol. Metab. 26, 803-807 (2013).

33. Derman, O., Kanbur, N., Kilic, I. \& Kutluk, T. Long-term follow-up of tamoxifen treatment in adolescents with gynecomastia. J. Pediatr. Endocrinol. Metab. 21, 449-454 (2008).

34. Eugster, E. A. et al. Tamoxifen treatment for precocious puberty in McCuneAlbright syndrome: a multicenter trial. J. Pediatr. 143, 60-66 (2003).

35. de, G. et al. Tamoxifen improves final height prediction in girls with McCuneAlbright syndrome: a long follow-up. Horm. Res. Paediatr. 84, 184-189 (2015).

36. Dorchies, O. M. et al. The anticancer drug tamoxifen counteracts the pathology in a mouse model of duchenne muscular dystrophy. Am. J. Pathol. 182, 485-504 (2013)

37. Gayi, E. et al. Repurposing the selective oestrogen receptor modulator tamoxifen for the treatment of Duchenne muscular dystrophy. Chimia $\mathbf{7 2}$, 238-240 (2018).

38. Buj-Bello, A. et al. The lipid phosphatase myotubularin is essential for skeletal muscle maintenance but not for myogenesis in mice. Proc. Natl Acad. Sci. USA 99, 15060-15065 (2002).

39. Al-Qusairi, L. et al. Lack of myotubularin (MTM1) leads to muscle hypotrophy through unbalanced regulation of the autophagy and ubiquitinproteasome pathways. Faseb J. 27, 3384-3394 (2013).

40. Sabha, N. et al. PIK3C2B inhibition improves function and prolongs survival in myotubular myopathy animal models. J. Clin. Invest. 126, 3613-3625 (2016).

41. Bevilacqua, J. A. et al. "Necklace" fibers, a new histological marker of lateonset MTM1-related centronuclear myopathy. Acta Neuropathol. 117, 283 (2008).

42. Cowling, B. S., Toussaint, A., Muller, J. \& Laporte, J. Defective membrane remodeling in neuromuscular diseases: Insights from animal models. PLoS Genet. 8, e1002595 (2012).

43. Laporte, J., Kress, W. \& Mandel, J. L. Diagnosis of X-linked myotubular myopathy by detection of myotubularin. Ann. Neurol. 50, $42-46$ (2001).

44. Dorchies, O. M. et al. Normal innervation and differentiation of X-linked myotubular myopathy muscle cells in a nerve-muscle coculture system. Neuromuscul. Disord. 11, 736-746 (2001).

45. Hnia, K. et al. Myotubularin controls desmin intermediate filament architecture and mitochondrial dynamics in human and mouse skeletal muscle. J. Clin. Invest. 121, 70-85 (2011).

46. Kerr, J. P., Ward, C. W. \& Bloch, R. J. Dysferlin at transverse tubules regulates $\mathrm{Ca}(2+)$ homeostasis in skeletal muscle. Front. Physiol. 5, 89 (2014).

47. Hofhuis, J. et al. Dysferlin mediates membrane tubulation and links T-tubule biogenesis to muscular dystrophy. J. Cell. Sci. 130, 841 (2017).

48. Hnia, K., Vaccari, I., Bolino, A. \& Laporte, J. Myotubularin phosphoinositide phosphatases: cellular functions and disease pathophysiology. Trends Mol. Med. 18, 317-327 (2012).

49. Lawlor, M. W. et al. Inhibition of activin receptor type IIB increases strength and lifespan in myotubularin-deficient mice. Am. J. Pathol. 178, 784-793 (2011).

50. Dowling, J. J. et al. Myotubular myopathy and the neuromuscular junction: a novel therapeutic approach from mouse models. Dis. Model Mech. 5, 852-859 (2012).

51. Robb, S. A. et al. Impaired neuromuscular transmission and response to acetylcholinesterase inhibitors in centronuclear myopathies. Neuromuscul. Disord. 21, 379-386 (2011)

52. Al-Qusairi, L. et al. T-tubule disorganization and defective excitationcontraction coupling in muscle fibers lacking myotubularin lipid phosphatase. Proc. Natl Acad. Sci. USA 106, 18763-18768 (2009).

53. Al-Qusairi, L. \& Laporte, J. T-tubule biogenesis and triad formation in skeletal muscle and implication in human diseases. Skelet. Muscle 1, 26-26 (2011).

54. Royer, B. et al. The myotubularin-amphiphysin 2 complex in membrane tubulation and centronuclear myopathies. EMBO Rep. 14, 907-915 (2013).

55. Kutchukian, C. et al. Phosphatidylinositol 3-kinase inhibition restores $\mathrm{Ca}^{2+}$ release defects and prolongs survival in myotubularin-deficient mice. Proc. Natl. Acad. Sci. USA 113, 14432-14437 (2016).

56. Vogel, V. G. et al. Effects of tamoxifen vs raloxifene on the risk of developing invasive breast cancer and other disease outcomes. JAMA 295, 2727-2741 (2006).

57. Van der Weijden-Van Doornik, E. M., Slot, D. E., Burtin, C. \& van der Weijden, G. A. Grip strength in women being treated for breast cancer and receiving adjuvant endocrine therapy: systematic review. Phys. Ther. 97, 904-914 (2017).

58. Baltgalvis, K. A., Greising, S. M., Warren, G. L. \& Lowe, D. A. Estrogen regulates estrogen receptors and antioxidant gene expression in mouse skeletal muscle. PLoS ONE 5, e10164 (2010).
59. Saceda, M. et al. Regulation of the estrogen receptor in MCF-7 cells by estradiol. Mol. Endocrinol. 2, 1157-1162 (1988).

60. Lonard, D. M., Nawaz, Z., Smith, C. L. \& O'Malley, B. W. The 26S proteasome is required for estrogen receptor-a and coactivator turnover and for efficient estrogen receptor-a transactivation. Mol. Cell 5, 939-948 (2000).

61. Fetalvero, K. M. et al. Defective autophagy and mTORC1 signaling in myotubularin null mice. Mol. Cell. Biol. 33, 98-110 (2013).

62. Totta, P., Busonero, C., Leone, S., Marino, M. \& Acconcia, F. Dynamin II is required for $17 \beta$-estradiol signaling and autophagy-based ERa degradation. Sci. Rep. 6, 23727 (2016).

63. Maani, N. et al. Tamoxifen therapy in a murine model of myotubular myopathy. Nat Commun. https://doi.org/41467-018-07057-5 (2018).

64. Sarnat, H. B. Myotubular myopathy: arrest of morphogenesis of myofibres associated with persistence of fetal vimentin and desmin. Four cases compared with fetal and neonatal muscle. Can. J. Neurol. Sci. 17, 109-123 (1990).

65. Demonbreun, A. R. \& McNally, E. M. Dynamin 2 the rescue for centronuclear myopathy. J. Clin. Invest. 124, 976-978 (2014).

66. Pemmaraju, N., Munsell, M. F., Hortobagyi, G. N. \& Giordano, S. H. Retrospective review of male breast cancer patients: analysis of tamoxifenrelated side-effects. Ann. Oncol. 23, 1471-1474 (2011).

67. Karatas, F. et al. Leg cramps associated with tamoxifen use-possible mechanism and treatment recommendations. J. Buon. 21, 520 (2016).

68. Lintermans, A. et al. A prospective assessment of musculoskeletal toxicity and loss of grip strength in breast cancer patients receiving adjuvant aromatase inhibitors and tamoxifen, and relation with BMI. Breast Cancer Res. Treat. 146, 109-116 (2014).

69. Bohm, J. et al. Altered splicing of the BIN1 muscle-specific exon in humans and dogs with highly progressive centronuclear myopathy. PLoS Genet. 9, e1003430 (2013).

70. Dorchies, O. M. et al. Green tea extract and its major polyphenol (-)-epigallocatechin gallate improve muscle function in a mouse model for Duchenne muscular dystrophy. Am. J. Physiol. Cell. Physiol. 290, C616-C625 (2006).

71. Hibaoui, Y., Reutenauer-Patte, J., Patthey-Vuadens, O., Ruegg, U. T. \& Dorchies, O. M. Melatonin improves muscle function of the dystrophic $\mathrm{mdx}^{5 \mathrm{Cv}}$ mouse, a model for Duchenne muscular dystrophy. J. Pineal Res. 51 , 163-171 (2011).

72. Reutenauer, J., Dorchies, O. M., Patthey-Vuadens, O., Vuagniaux, G. \& Ruegg U. T. Investigation of Debio 025, a cyclophilin inhibitor, in the dystrophic mdx mouse, a model for Duchenne muscular dystrophy. Br. J. Pharmacol. 155, 574-584 (2008).

73. Reutenauer-Patte, J., Boittin, F.-X., Patthey-Vuadens, O., Ruegg, U. T. \& Dorchies, O. M. Urocortins improve dystrophic skeletal muscle structure and function through both PKA- and Epac-dependent pathways. Am. J. Pathol. 180, 749-762 (2012).

74. De Andrade, P. B. M. et al. Caloric restriction induces energy-sparing alterations in skeletal muscle contraction, fiber composition and local thyroid hormone metabolism that persist during catch-up fat upon refeeding. Front. Physiol. 6, 254 (2015)

75. Cowling, B. S. et al. Increased expression of wild-type or a centronuclear myopathy mutant of dynamin 2 in skeletal muscle of adult mice leads to structural defects and muscle weakness. Am. J. Pathol. 178, 2224-2235 (2011).

76. Amoasii, L. et al. Phosphatase-dead myotubularin ameliorates X-linked centronuclear myopathy phenotypes in mice. PLoS Genet. 8, e1002965 (2012).

77. Boittin, F.-X. et al. $\mathrm{Ca}^{2+}$-independent phospholipase $\mathrm{A}_{2}$ enhances storeoperated $\mathrm{Ca}^{2+}$ entry in dystrophic skeletal muscle fibers. J. Cell. Sci. 119, 3733-3742 (2006)

\section{Acknowledgements}

This work was supported by intramural research support from the University of Geneva, University of Lausanne and University of Strasbourg, as well as by grants to O.M.D. and L.S. from the Swiss Foundation for Research on Muscle Diseases (SFRMD/FSRMM/ SSEM), the French Muscular Dystrophy Association (AFM-Telethon; Grants \#17821, \#20959), and the Dutch Duchenne Parent Project (DPP-NL) and grants to B.S.C. and J.L. from INSERM, CNRS, Agence Nationale de la Recherche (ANR-14-CE12-0009, -10LABX-0030-INRT and -10-IDEX-0002-02). M.S. is supported by the European Union's Horizon 2020 research and innovation programme under the Marie Sklodowska-Curie grant agreement No 800198. X.M.M. is an IGBMC International PhD Programme fellow supported by LabEx INRT funds. We are grateful to Colette Sauty for excellent assistance with animal care, Barbara Pinheiro-Tonneau and Stefane Melancia for help with histological analyses, Pascal Kessler for help with image quantification and Nadia Messaddeq for help with electron microscopy.

\section{Author contributions}

E.G., L.A.N., X.M.M., H.M.I., M.S., T.M., B.S.C., and O.M.D. designed and performed the experiments, E.G., L.A.N., X.M.M., H.M.I., M.S., T.M., B.S.C., and O.M.D. analyzed the data; E.G., L.A.N., H.M.I., and O.M.D. wrote the manuscript; X.M.M., M.S., T.M., L.A.D., 
J.L., B.S.C., and L.S. edited the manuscript; L.A.D., J.L., B.S.C., L.S., and O.M.D. provided support and supervised the work.

\section{Additional information}

Supplementary Information accompanies this paper at https://doi.org/10.1038/s41467018-07058-4.

Competing interests: J.L. and B.S.C. are scientific advisors for Dynacure. The remaining authors declare no competing interests.

Reprints and permission information is available online at http://npg.nature.com/ reprintsandpermissions/ (c) (i) Open Access This article is licensed under a Creative Commons Attribution 4.0 International License, which permits use, sharing, adaptation, distribution and reproduction in any medium or format, as long as you give appropriate credit to the original author(s) and the source, provide a link to the Creative Commons license, and indicate if changes were made. The images or other third party material in this article are included in the article's Creative Commons license, unless indicated otherwise in a credit line to the material. If material is not included in the article's Creative Commons license and your intended use is not permitted by statutory regulation or exceeds the permitted use, you will need to obtain permission directly from the copyright holder. To view a copy of this license, visit http://creativecommons.org/ licenses/by/4.0/.

(C) The Author(s) 2018

Publisher's note: Springer Nature remains neutral with regard to jurisdictional claims in published maps and institutional affiliations. 